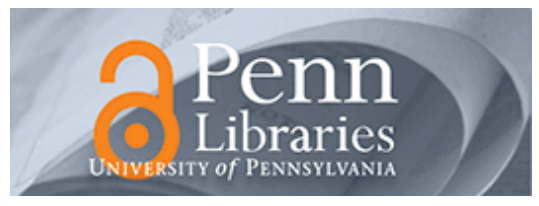

University of Pennsylvania

ScholarlyCommons

Marketing Papers

Wharton Faculty Research

$1-2016$

\title{
Participation vs. Effectiveness of Paid Endorsers in Social Advertising Campaigns: A Field Experiment
}

Jing Peng

University of Pennsylvania

Christophe Van den Bulte

University of Pennsylvania

Follow this and additional works at: https://repository.upenn.edu/marketing_papers

Part of the Advertising and Promotion Management Commons, Applied Behavior Analysis Commons, Behavioral Economics Commons, Marketing Commons, Mass Communication Commons, and the Social Influence and Political Communication Commons

\section{Recommended Citation}

Peng, J., \& Bulte, C. (2016). Participation vs. Effectiveness of Paid Endorsers in Social Advertising Campaigns: A Field Experiment. http://dx.doi.org/10.2139/ssrn.2702053

This is an unpublished version.

This paper is posted at ScholarlyCommons. https://repository.upenn.edu/marketing_papers/377

For more information, please contact repository@pobox.upenn.edu. 


\title{
Participation vs. Effectiveness of Paid Endorsers in Social Advertising Campaigns: A Field Experiment
}

\author{
Abstract \\ We investigate the participation and effectiveness of paid endorsers in viral-for-hire social advertising. We \\ conduct a field experiment with an invitation design in which we manipulate both incentives and a soft \\ eligibility requirement to participate in campaigns. The latter provides a strong and valid instrument to \\ separate participation from outcomes effects. Since likes, comments, and retweets are count variables, \\ and since potential endorsers can self-select to participate in multiple campaigns, we propose a Poisson \\ lognormal model with sample selection and correlated random effects to analyze variations in \\ participation and effectiveness. There are three main findings. (1) Payments higher than the average \\ reward a potential endorser received in the past (gains) do not increase participation, whereas lower \\ payments (losses) decrease participation. Neither gains nor losses affect effectiveness. (2) Potential \\ endorsers who are more likely to participate tend to be less effective. (3) Which endorser characteristics \\ are associated with effectiveness depends on whether success is measured in likes, comments, or \\ retweets. These findings provide new insights on how marketers can improve social advertising \\ campaigns by better targeting and incenting potential endorsers.
}

\section{Keywords}

paid endorsement, social advertising, targeting, viral marketing, sample selection

\section{Disciplines}

Advertising and Promotion Management | Applied Behavior Analysis | Behavioral Economics | Business | Marketing | Mass Communication | Social Influence and Political Communication

\section{Comments}

This is an unpublished version. 


\title{
Participation vs. Effectiveness of Paid Endorsers in Social Advertising Campaigns: A Field Experiment
}

\author{
Jing Peng and Christophe Van den Bulte \\ The Wharton School, University of Pennsylvania \\ \{jingpeng, vdbulte\}@wharton.upenn.edu
}

January 15, 2016

Acknowledgments. We benefited from feedback from session participants at the 2015 Marketing Science Conference, the 2015 Workshop on Information in Networks, the 2015 INFORMS Annual Meeting, and the 2015 International Conference on Information Systems. This project was made possible by financial support extended to the first author through a Penn Lauder CIBER PhD Grant and a Wharton Baker Retailing Center PhD Research Grant. 


\title{
Participation vs. Effectiveness of Paid Endorsers in Social Advertising Campaigns: A Field Experiment
}

\author{
Jing Peng and Christophe Van den Bulte \\ The Wharton School, University of Pennsylvania \\ \{jingpeng, vdbulte\}@wharton.upenn.edu
}

\begin{abstract}
We investigate the participation and effectiveness of paid endorsers in viral-for-hire social advertising. We conduct a field experiment with an invitation design in which we manipulate both incentives and a soft eligibility requirement to participate in campaigns. The latter provides a strong and valid instrument to separate participation from outcomes effects. Since likes, comments, and retweets are count variables, and since potential endorsers can self-select to participate in multiple campaigns, we propose a Poisson lognormal model with sample selection and correlated random effects to analyze variations in participation and effectiveness. There are three main findings. (1) Payments higher than the average reward a potential endorser received in the past (gains) do not increase participation, whereas lower payments (losses) decrease participation. Neither gains nor losses affect effectiveness. (2) Potential endorsers who are more likely to participate tend to be less effective. (3) Which endorser characteristics are associated with effectiveness depends on whether success is measured in likes, comments, or retweets. These findings provide new insights on how marketers can improve social advertising campaigns by better targeting and incenting potential endorsers.
\end{abstract}

Keywords: Paid endorsement, Social advertising, Targeting, Viral marketing, Sample selection. 


\section{Introduction}

Social advertising leverages social connections among consumers to reach and influence a target audience. This business practice is becoming increasingly popular. According to BI intelligence ${ }^{1}$, social advertising spending in the US will top $\$ 8.5$ billion in 2015 and reach nearly $\$ 14$ billion by 2018 . Globally, it is expected to reach $\$ 23.7$ billion in 2015 and $\$ 36$ billion by 2017, capturing 16\% share of all digital ad spending ${ }^{2}$. Two thirds of marketers believe that social media is core to their business, and $70 \%$ of them plan to increase the budget on social media marketing ${ }^{3}$.

The prevalent social advertising mechanism is a centralized system in which advertisers submit ads to social media platforms (publishers) who then decide how to distribute the ads. Two drawbacks of this system are that advertisers have no direct control over the selection of endorsers (e.g., users who share/retweet an ad on Facebook/Twitter) and that endorsers are not incentivized to get engaged. Paid endorsement, in contrast, is a decentralized mechanism that allows advertisers to bypass publishers and recruit individual endorsers of their own choice at pre-specified prices. Specifically, advertisers post tasks on a paid endorsement platform (a broker website similar to Amazon Mechanical Turk) and microbloggers registered on the platform can take on the tasks requiring them to post or retweet some ad for monetary rewards. Paid endorsement has gained particular popularity in China, with many websites acting as platforms for paid endorsement. Weibo.com, the largest Chinese microblog site with more than 500 million users, launched its official paid endorsement platform in 2012.

Despite the growing interest in paid endorsement and social advertising in general, its effectiveness remains in question. Two thirds of advertisers are uncertain about the effectiveness of social advertising ${ }^{4 \& 5}$. The effectiveness of a paid endorsement campaign depends on how many endorsers participate and on how well they expand reach (i.e., views), generate engagement (i.e., likes, comments, and retweets), increase traffic (i.e., clicks), and boost sales.

A key question facing marketers is how to incent endorsers. One problem with paid endorsement is that the incentive of participants is contingent on participation rather than performance, as monitoring performance is often practically infeasible or too costly. So far, the incentive on most paid endorsement platforms (e.g., weituitui.com and sandaha.com) are simply determined by the number of followers an endorser has. Research on survey response behavior shows that incentives unrelated to performance typically increase participation, but rarely affect performance (Cantor et al. 2008; Singer and Ye 2013). The same may hold in paid endorsement campaigns. A second source of complexity is that the reaction to a

\footnotetext{
${ }^{1}$ http://www.businessinsider.com/social-media-advertising-spending-growth-2014-9

${ }^{2} \mathrm{http}$ ///www.emarketer.com/Article/Social-Network-Ad-Spending-Hit-2368-Billion-Worldwide-2015/1012357

${ }^{3}$ http://www.adweek.com/socialtimes/social-marketing-2015/504357

${ }^{4} \mathrm{http}: / /$ www.nielsen.com/content/dam/corporate/us/en/reports-downloads/2013\%20Reports/Nielsen-Paid-Social-Media-Adv-Report-2013.pdf

${ }^{5}$ http://www.socialmediaexaminer.com/SocialMediaMarketingIndustryReport2014.pdf
} 
particular level of financial incentive is likely to vary across potential endorsers with different award histories, as implied by prospect theory positing reference dependence and loss aversion, two phenomena well-documented to affect consumer behavior (Greenleaf 1995; Hardie et al. 1993; Kalyanaram and Winer 1995; Lattin and Bucklin 1989).

A second key question is which endorsers to target. Whether or not an endorser is worth targeting not only depends on the endorser's effectiveness in generating desired outcomes (e.g., engagements and sales), but also the endorsers' willingness to participate, as only participants can generate real outcomes. To design successful targeting strategies, it's critical for marketers to understand which endorsers are responsive (in participation) and which endorsers are effective (in generating outcomes), and more importantly, whether responsive endorsers are also effective. Meanwhile, given that different marketers may have different objectives in their campaigns, whether the effectiveness of endorsers varies across different types of outcomes might be also of interest to marketers.

Customer engagement in the form of likes, comments, and retweets is a key objective to marketers and can easily be tracked at the endorser level. Several studies have already investigated how characteristics of online users are associated with their influence on others (Aral and Walker 2012; Katona et al. 2011; Trusov et al. 2010). However, these studies concentrate on organic word of mouth and voluntary endorsement without monetary incentive (Shi et al. 2014; Toubia and Stephen 2013). Their findings need not generalize to paid endorsement campaigns with monetary incentives. For instance, self-presentation is often a key motive to post online content (Schau and Gilly 2003; Toubia and Stephen 2013), but it is not clear to what extent this holds in paid endorsement and other viral-for-hire campaigns.

This paper aims at filling in this gap in the literature by providing answers to the following questions: (i) how incentive affect endorsers' participation and effectiveness in paid endorsement campaigns, (ii) what types of endorsers are most effective in generating online engagements, and (iii) whether that varies across types of engagements that require different levels of effort from endorsers' followers.

To answer these questions, we collaborated with two vendors on the Chinese retailing site taobao.com, and ran a field experiment on the Chinese microblogging site weibo.com, using one of the largest Chinese paid endorsement platforms, weituitui.com. We exogenously manipulate the pay rate to endorsers and their eligibility to participate. Since the data collected from our experiment are panel count data with sample selection issues, we propose a Poisson lognormal model with sample selection and correlated random effects to analyze what affects endorsers' participation and effectiveness.

Our study produces several intriguing findings. (i) Endorsers are sensitive to losses but not gains, compared to the average reward per task they received in the past. (ii) Observed and unobserved characteristics of endorsers often have opposite effects on participation and effectiveness. As a result, low potential endorsers may generate high actual engagements due to their high probability to participate, 
whereas high potential endorsers may generate low actual engagements due to their low probability to participate. (iii) The potential of the same endorser can be different in generating different types of engagement.

This work, as the first attempt to study what affects endorsers' participation and effectiveness in paid endorsement social advertising campaigns, makes the following contributions to the literature. First, it helps marketers understand the role of incentives in such campaigns. Second, it documents a tension between participation and effectiveness, and highlights the difference between potential and actual effectiveness. Third, it suggests that different mechanisms may be driving different types of engagements. Finally, it shows how to deal with sample selection in panel data with repeated observations by combining an exogenous soft eligibility constraint and econometric modeling.

\section{Theoretical Background}

This section discusses motives that may affect endorsers' participation and effectiveness in paid endorsement campaigns, and how financial incentives and three endorsers' characteristics (social media fan base, prior activity level, and community embeddedness) may affect endorsers' participation and effectiveness.

\subsection{Participation}

The literature on survey participation broadly divides the reasons why people participate in surveys or questionnaires into three categories: altruistic reasons (e.g., willingness to help research and civil duty), egoistic reasons (e.g., monetary incentive, opportunity to learn something), and survey-specific reasons (e.g., topical interest, trust in organization) (Singer and Ye 2013). Likewise, in paid endorsement campaigns, the motives of endorsers can be classified into three categories: altruistic (e.g., goodwill to share attractive deals), egoistic (e.g., monetary incentive and self-enhancement), and campaign-specific. In this paper, we only focus on drivers that are relevant to incentive and endorsers' characteristics. Two such drivers are monetary incentive and self-enhancement (perhaps also goodwill to share attractive deals, which is hard to disentangle with self-enhancement).

Incentives, reference dependence and loss aversion. Paid endorsement is predicated on the assumption that financial incentives motivate people to act as endorser. Prospect theory posits that financial rewards motivate people because they are gains or losses compared to some reference point, rather than merely because of the absolute size of the incentive (Kahneman and Tversky 1979; Long and Nasiry 2015). It also posits that people are often more sensitive to losses than gains. The former phenomenon is known as reference dependence and the latter as loss aversion. Both are well-documented in consumer behavior (Greenleaf 1995; Hardie et al. 1993; Kalyanaram and Winer 1995; Lattin and Bucklin 1989). Prior research suggests that the average reward per task that a potential endorser received in the past is a good candidate 
reference point in paid endorsement campaigns (e.g., Hardie et al. 1993). Both the theory and empirical findings, finally, indicate the presence of deceasing rather than constant returns in how gains and losses affect behavior.

Self-enhancement. Theories of self-enhancement suggest that people are motivated to seek positive evaluations from others (Jones 1973). On social media platforms, users' activities are publicly visible to others. This makes social media platforms an ideal place for people to signal their expertise and enhance their social status (Alexandrov et al. 2013; Lovett et al. 2013; Schau and Gilly 2003). In particular, Toubia and Stephen (Toubia and Stephen 2013) documented that self-image is the primary motive for most users to contribute content voluntarily to Twitter. Therefore, users with a positive reputation and self-image may be more selective than others in which paid endorsement campaigns to participate. The concern about selfenhancement likely varies with several characteristics of endorsers, as elaborated below.

Social media fan base refers to the number of followers that endorsers on social media platforms have. Since the remuneration of endorsers often increases with their number of followers, endorsers with a larger number of followers might be financially more motivated to participate. However, users with a larger number of followers may derive more self-image related utility (Toubia and Stephen 2013). As a result, they might be more selective about which campaigns to participate in, as broadcasting irrelevant content can hurt their reputation (Barasch and Berger 2014; Bock et al. 2005). Alternatively, it is possible that endorsers with a greater number of followers are more likely to participate regardless of incentive, as they derive more intrinsic and status-related benefits from relaying attractive deals and other interesting content (Toubia and Stephen 2013).

Prior activity level refers to the endorsers' past activity intensity on social media and paid endorsement platforms. The more posts a user made on social media, and the more paid endorsement campaigns a user participated in, the less selective the user may be in deciding what to post and what to participate in (compare Porter and Whitcomb 2003). Therefore, we expect endorsers who posted more and participated more in the past to be more likely to participate in a future campaign.

Community embeddedness refers to how long the endorsers have been registered and how many friends they have in the paid endorsement community. Endorsers who are more deeply embedded into the community might be more selective in what campaigns to participate in (Minkler 2012), and more concerned about their status when sharing content in online communities (Schau and Gilly 2003; Toubia and Stephen 2013). Thus, such endorsers may be more selective and less likely to participate in any given endorsement campaign.

\subsection{Effectiveness}

The effectiveness of endorsers in generating engagements depends on their level of effort, the trust of their followers in them, the sheer numbers of followers, and the strength of the ties with their followers 
(e.g., Aral and Walker 2014; Chu and Kim 2011; King et al. 2014; Moldoveanu and Baum 2011). We discuss the potential effects of incentive size and the three types of endorser characteristics we study based on how they relate to these four traits.

Incentive. In paid endorsement platforms, the remuneration of endorsers is often based solely on their number of followers rather than being contingent on performance. Research on survey response behavior suggest two alternative hypotheses regarding the impact of incentive on response quality when incentives do not depend on performance (Cantor et al. 2008; Singer and Ye 2013). One hypothesis is that, by attracting people who would otherwise not participate, the quality of response declines. The alternative hypothesis is that, by rewarding participants, the quality of responses increases due to feelings of gratitude or obligation. A comprehensive review of studies evaluating the effects of incentive on response quality (e.g., number of questions answered and length of answers) concluded that incentive size almost never had an effect on quality (Singer and Ye 2013). This suggests that in paid endorsement campaigns, the size of incentive need not impact the effectiveness of endorsers. Therefore, we expect little to no effect of incentive on effectiveness.

Social media fan base. While the tie strength between users and their contacts decreases with the number of contacts (Burke 2011; Katona et al. 2011; Roberts et al. 2009), a larger fan base implies a larger audience who can potentially engage (Goel et al. 2015). A number of studies have investigated the effect of network size on a user's overall influence, but the results are mixed. Katona et al. (2011) find that the effectiveness of individuals in influencing friends to adopt (register on) a social network site decreases with the total number of their contacts, whereas Yoganarasimhan (2012) finds that a node's overall effectiveness in spreading Youtube videos increases with its network size. One explanation to reconcile these two findings is that the effect of network size depends on the level of effort needed to make a decision. When the required effort is small (e.g., information diffusion), weak ties suffice (Granovetter 1973; Weimann 1983 ) and the effect of network size is dominated by volume per se, leading to a positive overall effect. On the other hand, when the required effort is large (e.g., product adoption), the need for strong ties (Weenig and Midden 1991; Weimann 1983) make users with larger number of followers connected by weak ties not as persuasive, resulting in a negative overall effect. This implies that the effect of the number of weak tie followers on comments and retweets might be smaller than that on likes, as comments and retweets require more effort than likes.

Priority activity level. Endorsers who posted and participated a lot in the past are less likely to be selective and more likely to be spammers. Numerous posts or endorsements can hurt their reputation, rendering them less trustworthy than those who do not post/endorse as much (Barasch and Berger 2014; Bock et al. 2005). Therefore, endorsers who posted and participated more in the past should be less effective. 
Note, this implies that endorser characteristics associated with prior activity may have opposite effects on participation and effectiveness.

Community embeddedness. Following the argument that endorsers who are embedded into the paid endorsement community tend to be more selective in what to participate, it is likely that their follower will trust their endorsements more. Consequently, endorsers with stronger community embeddedness are expected to be more effective in generating online engagements from their followers. Note, this implies that endorser characteristics associated with community embeddedness may have opposite effects in participation and effectiveness.

\section{Field Experiment}

\subsection{Research Setting}

We conducted a field experiment on weituitui.com, a social advertising platform with more than 40,000 registered endorsers who own accounts on weibo.com. Weituitui.com is a broker website that allows advertisers to recruit endorsers at pre-specified prices for their social media marketing campaigns. An advertiser can initiate a paid endorsement campaign by posting a task describing her needs on weituitui.com. In the task, the advertiser also specifies how much an endorser will be paid, as a linear or step-wise linear function of the endorser's number of followers on weibo.com. To penalize robot followers and inactive followers, weituitui.com uses the number of verified followers to calculate the reward for an endorser. Weituitui.com has an internal algorithm to compute the percentage of verified followers based on how actively an endorser's followers engage on her past tweets. Similar to other paid endorsement platforms like sandaha.com, weituitui.com has several policies in place to make sure that the rewards are not too small to be meaningful and also to encourage endorsers with small numbers of followers to participate. The rewards for endorsers with less than 1000 verified followers are fixed on weituitui.com (10-49: 0.1RMB, 50-99: 0.2RMB, 100-499: 0.3RMB, 500-999: 0.5RMB), regardless of the reward structure. Endorsers with less than 10 verified followers are not allowed to participate. The reward for endorsers with more than 1000 verified followers are no less than 0.5RMB.

In a task, the advertiser provides the URL of the target tweet containing the product information. The advertiser can impose some written requirements for the task, such as how long the endorser should keep (i.e., not delete) the retweet on their timeline, and the minimal length of the comment in the retweet. Furthermore, the advertiser can specify who is eligible for the task. Some eligibility restrictions are hard restrictions automated by the platform, such as the allowable day part of participation (e.g., 9am-9pm), while other are soft restrictions attached in the written requirements that need to be manually verified afterwards. If an endorser decides to participate, she needs to retweet the given tweet, fulfill the requirements, and then submit the URL of her retweet. The duration of a task ranges from 3 to 5 days. Once 
the task ends, the advertiser has 3 days to manually approve or disapprove the submissions, depending on whether the endorser has truly retweeted the given tweet and fulfilled the requirements. All remaining submissions are approved automatically by the platform after the 3-day window. Because of this autoapproval policy, opportunistic endorsers or spammers may submit a random URL even if they haven't retweeted the tweet. For approved tasks, the endorsers are paid and weituitui charges a $30 \%$ commission fee. That fee is reduced to $15 \%$ for an extremely small proportion $(0.3 \%)$ of VIP endorsers who have spent (rather than earned) more than 1000RMB on weituitui.com.

\subsection{Experiment Design}

To investigate the effect of incentive on endorsers' willingness to participate and their effectiveness in generating engagements (i.e., likes, comments, and retweets), we exogenously manipulate the incentive by posting two identical tasks at two different pay rates. We use the linear pricing scheme as it is easier to implement and understand. The two pay rates are $0.0002 \mathrm{RMB}(1 \mathrm{RMB} \approx 0.16 \mathrm{USD})$ and $0.0004 \mathrm{RMB}$ per follower, respectively. The former is the lowest possible and most common rate for linear pricing (i.e., $87 \%$ of tasks $)^{6}$, whereas the latter is higher than or equal to $96 \%$ of linear rates used on weituitui.com. Figure 1 plots the incentive curves for the two pay rates, showing how the number of verified followers maps into the financial rewards at the low and high pay rates. The percentile of endorsers is given on the top of the figure (e.g., 59\% endorsers have less than 500 verified followers). Note how rewards at different pay rates differ for only about $23 \%$ of endorsers with the most verified followers.

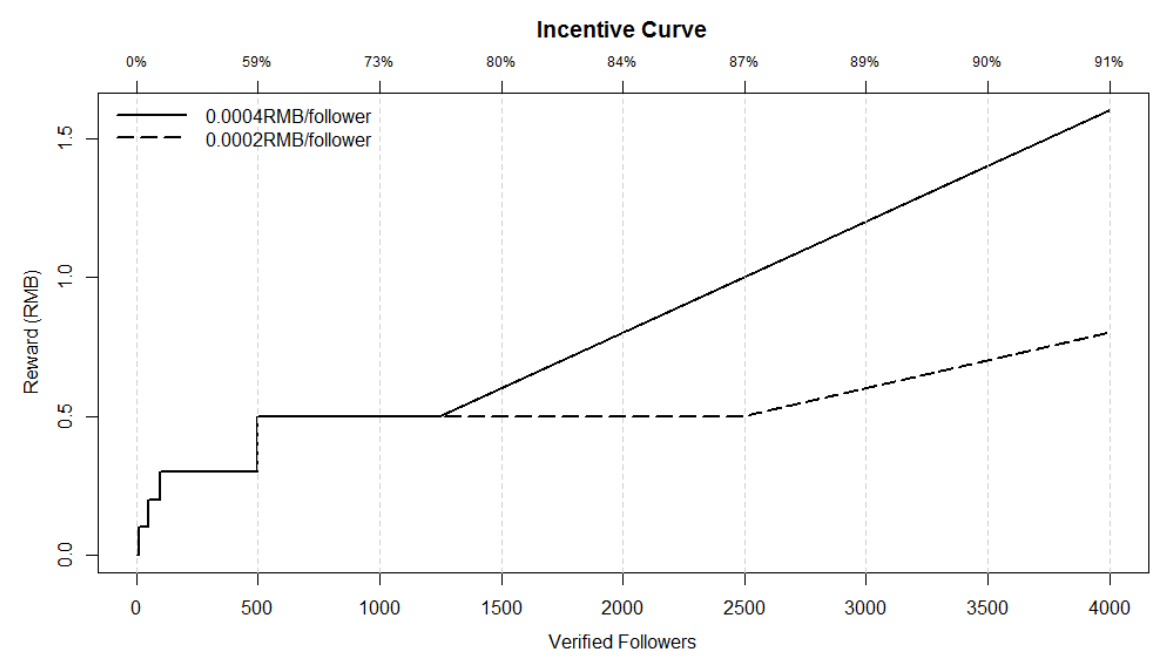

Figure 1. The incentive curves at low and high pay rates.

To make sure that the two tasks are indeed identical and yet independent with each other, we register a new account on weibo.com and post two identical tweets on the same product at roughly the same time

${ }^{6}$ Historically, $1 \%$ of linearly priced tasks were posted at the rate of 0.00015 RMB per follower, but the minimum rate had been changed to 0.0002 RMB per follower more than two years before we ran the experiment. 
(more precisely, one is posted just seconds ahead of the other). The URLs of the two tweets are then used in the two tasks, respectively. Since the new account has no followers, all the observed engagements on the two tweets come from the paid endorsers and their followers. To eliminate the potential effects resulting from the order of the two tweets, the pay rates associated with the tasks posted first and second are swapped from time to time.

Since there might be unobserved variables that affect both participation and effectiveness of endorsers, the identification of the effects of endorser characteristics in both stages typically requires an exclusion restriction (Puhani 2000). To that end, we add a soft eligibility restriction in our tasks, such that every endorser on weituitui.com is only eligible for one of the two identical tasks. "Soft" means that ineligible endorsers can still participate, but will not be paid. This is known as an invitation or encouragement design (Brewer 1976; Duflo and Saez 2003; Powers and Swinton 1984). Specifically, endorsers whose last two digits of their weituitui IDs (six-digit numbers) are among a certain range are eligible to participate in one task, and those among another range are eligible for the other task. The eligibility restriction is a valid instrument as the last two digits of an endorser's ID are random and unrelated to her effectiveness.

Our experiment was conducted in 8 different weeks between 2/1/2014 and 4/26/2014. Each week, we posted two groups (pairs) of identical tasks on two products from the same vendor. Accordingly, we divided endorsers into 4 different groups based on their ID (i.e., 00 24, 25 49, 50 74, 75 99), such that any endorser was eligible for only one of the four tasks in that week. The four tasks were posted simultaneously so that they showed up right next to each other. The tasks were rotated over 6 products from 2 vendors on taobao.com. Each task was open for participation for 72 hours. For our experiment, we did not impose any particular effort-related task requirements except for retweeting and liking the tweet. The eligible pay rates for the same endorsers were rotated across weeks. Table 1 visualizes the key conditions of our experimental design by showing the four tasks posted in a given week. Each task pertains to one of two products for which endorsers are promised either a high or low pay rate, and a potential endorser qualifies for only one of the four tasks.

Table 1. Experimental Design

\begin{tabular}{|l|l|l|}
\hline & Pair A & Pair B \\
\hline Price: $0.0002 \mathrm{RMB} /$ follower & $00 \sim 24$ & $50 \sim 74$ \\
\hline Price: $0.0004 \mathrm{RMB} /$ follower & $25 \sim 49$ & $75 \sim 99$ \\
\hline
\end{tabular}

Eligible IDs shown in cells were rotated across pay rates and products across weeks.

\section{Data}

\subsection{Descriptive Statistics}

The data is collected and analyzed at the endorser level. We focus on the 8,283 active endorsers who participated in at least one paid endorsement task in the 6 months prior to our experiment. In every task, we 
record whether each of these active endorsers participates and how many engagements she generates. The number of engagements is collected for each retweeter/endorser using the API provided by weibo.com. Herein, participation means that an endorser has actually retweeted the message. The participation and engagement statistics are summarized in Table 2. Excluding one task for which we failed to track the engagement due to a technical issue, the 31 tasks we posted attracted 2,241 participations from 1,016 endorsers.

Table 2. Experiment Statistics

\begin{tabular}{|l|l|}
\hline Number of weeks & 8 \\
\hline Number of products & 6 \\
\hline Number of tasks & 31 \\
\hline Number of endorsers & 8,283 \\
\hline Number of participating endorsers & 1,016 \\
\hline Number of participations & 2,241 \\
\hline Number of participations from ineligible endorsers & 91 \\
\hline Number of <task, endorser> observations & 227,608 \\
\hline
\end{tabular}

Note that some endorsers registered on weituitui.com in the middle of our experiment

Table 3. Participation and Engagement Statistics of Tasks

\begin{tabular}{|c|c|c|c|c|c|c|c|c|c|c|c|c|c|c|c|c|}
\hline \multirow{2}{*}{ Week } & \multirow{2}{*}{ Pair } & \multirow{2}{*}{ Task } & \multirow{2}{*}{ Budget } & \multirow{2}{*}{$\begin{array}{l}\text { Pay } \\
\text { Rate }\end{array}$} & \multirow{2}{*}{ Product } & \multirow{2}{*}{$\begin{array}{c}\# \\
\text { Participants }\end{array}$} & \multirow{2}{*}{ \#Followers } & \multicolumn{3}{|c|}{ Total Engagements } & \multicolumn{3}{|c|}{ Average Engagements } & \multicolumn{3}{|c|}{ Maximum Engagements } \\
\hline & & & & & & & & likes & comments & retweets & likes & comments & retweets & likes & comments & retweets \\
\hline \multirow{4}{*}{1} & 1 & 1 & 100 & 4 & FT & 32 & $0.6 \mathrm{M}$ & 9 & 13 & 21 & 0.28 & 0.41 & 0.66 & 3 & 6 & 8 \\
\hline & 1 & 2 & 100 & 2 & FT & 3 & $1.3 \mathrm{M}$ & 8 & 67 & 67 & 2.67 & 22.33 & 22.33 & 8 & 34 & 36 \\
\hline & 2 & 3 & 100 & 4 & HRM & 34 & $0.6 \mathrm{M}$ & 17 & 5 & 5 & 0.50 & 0.15 & 0.15 & 15 & 4 & 4 \\
\hline & 2 & 4 & 100 & 2 & HRM & 65 & $0.8 \mathrm{M}$ & 11 & 120 & 121 & 0.17 & 1.85 & 1.86 & 4 & 21 & 23 \\
\hline \multirow{4}{*}{2} & 3 & 5 & 200 & 2 & ST & 113 & $1.4 \mathrm{M}$ & 8 & 40 & 46 & 0.07 & 0.35 & 0.41 & 1 & 12 & 14 \\
\hline & 3 & 6 & 200 & 4 & ST & 109 & $2.0 \mathrm{M}$ & 12 & 24 & 26 & 0.11 & 0.22 & 0.24 & 2 & 14 & 15 \\
\hline & 4 & 7 & 200 & 2 & BL & 91 & $3.6 \mathrm{M}$ & 9 & 14 & 18 & 0.10 & 0.15 & 0.20 & 1 & 6 & 6 \\
\hline & 4 & 8 & 200 & 4 & BL & 119 & $2.1 \mathrm{M}$ & 15 & 26 & 33 & 0.13 & 0.22 & 0.28 & 1 & 6 & 14 \\
\hline \multirow{4}{*}{3} & 5 & 9 & 300 & 4 & BL & 71 & $2.0 \mathrm{M}$ & 8 & 10 & 10 & 0.11 & 0.14 & 0.14 & 1 & 2 & 4 \\
\hline & 5 & 10 & 300 & 2 & BL & 84 & $2.8 \mathrm{M}$ & 4 & 20 & 34 & 0.05 & 0.24 & 0.40 & 2 & 7 & 20 \\
\hline & 6 & 11 & 300 & 4 & ST & 80 & $1.1 \mathrm{M}$ & 4 & 49 & 50 & 0.05 & 0.61 & 0.63 & 2 & 13 & 15 \\
\hline & 6 & 12 & 300 & 2 & ST & 78 & $2.2 \mathrm{M}$ & 6 & 14 & 7 & 0.08 & 0.18 & 0.09 & 1 & 4 & 5 \\
\hline \multirow{4}{*}{4} & 7 & 13 & 300 & 4 & ST & 56 & $0.8 \mathrm{M}$ & 3 & 2 & 5 & 0.05 & 0.04 & 0.09 & 1 & 2 & 4 \\
\hline & 7 & 14 & 300 & 2 & ST & 66 & $2.5 \mathrm{M}$ & 6 & 5 & 3 & 0.09 & 0.08 & 0.05 & 2 & 3 & 2 \\
\hline & 8 & 15 & 300 & 4 & BL & 81 & $1.4 \mathrm{M}$ & 6 & 25 & 32 & 0.07 & 0.31 & 0.40 & 2 & 10 & 12 \\
\hline & 8 & 16 & 300 & 2 & BL & 74 & $1.2 \mathrm{M}$ & 4 & 8 & 1 & 0.05 & 0.11 & 0.01 & 1 & 5 & 1 \\
\hline \multirow{4}{*}{5} & 9 & 17 & 200 & 2 & LP & 63 & $1.0 \mathrm{M}$ & 13 & 1 & 5 & 0.21 & 0.02 & 0.08 & 4 & 1 & 2 \\
\hline & 9 & 18 & 200 & 4 & LP & 77 & $0.7 \mathrm{M}$ & 8 & 3 & 0 & 0.10 & 0.04 & 0.00 & 3 & 3 & 0 \\
\hline & 10 & 19 & 200 & 2 & ER & 76 & $1.8 \mathrm{M}$ & 8 & 1 & 4 & 0.11 & 0.01 & 0.05 & 3 & 1 & 1 \\
\hline & 10 & 20 & 200 & 4 & ER & 70 & $1.3 \mathrm{M}$ & 0 & 1 & 2 & 0.00 & 0.01 & 0.03 & 0 & 1 & 1 \\
\hline \multirow{3}{*}{6} & 11 & 21 & 200 & 4 & HRM & 67 & $1.3 \mathrm{M}$ & 6 & 4 & 0 & 0.09 & 0.06 & 0.00 & 3 & 2 & 0 \\
\hline & 11 & 22 & 200 & 2 & HRM & 63 & $0.5 \mathrm{M}$ & 11 & 5 & 1 & 0.17 & 0.08 & 0.02 & 4 & 3 & 1 \\
\hline & 12 & 24 & 200 & 2 & FT & 70 & $1.2 \mathrm{M}$ & 2 & 1 & 1 & 0.03 & 0.01 & 0.01 & 1 & 1 & 1 \\
\hline \multirow{4}{*}{7} & 13 & 25 & 200 & 2 & ER & 72 & $1.1 \mathrm{M}$ & 8 & 3 & 1 & 0.11 & 0.04 & 0.01 & 2 & 1 & 1 \\
\hline & 13 & 26 & 200 & 4 & ER & 70 & $0.7 \mathrm{M}$ & 4 & 3 & 1 & 0.06 & 0.04 & 0.01 & 2 & 1 & 1 \\
\hline & 14 & 27 & 200 & 2 & LP & 80 & $1.5 \mathrm{M}$ & 9 & 8 & 2 & 0.11 & 0.10 & 0.03 & 1 & 2 & 1 \\
\hline & 14 & 28 & 200 & 4 & LP & 75 & $1.7 \mathrm{M}$ & 2 & 4 & 3 & 0.03 & 0.05 & 0.04 & 1 & 1 & 1 \\
\hline \multirow{4}{*}{8} & 15 & 29 & 200 & 4 & FT & 72 & $1.1 \mathrm{M}$ & 6 & 1 & 0 & 0.08 & 0.01 & 0.00 & 2 & 1 & 0 \\
\hline & 15 & 30 & 200 & 2 & FT & 79 & $0.9 \mathrm{M}$ & 3 & 3 & 2 & 0.04 & 0.04 & 0.03 & 1 & 1 & 1 \\
\hline & 16 & 31 & 200 & 4 & HRM & 81 & $1.6 \mathrm{M}$ & 9 & 5 & 6 & 0.11 & 0.06 & 0.07 & 2 & 1 & 6 \\
\hline & 16 & 32 & 200 & 2 & HRM & 70 & $1.4 \mathrm{M}$ & 5 & 9 & 0 & 0.07 & 0.13 & 0.00 & 1 & 8 & 0 \\
\hline
\end{tabular}

* For pay rate, "2" and " 4 " represent 0.0002 and $0.0004 \mathrm{RMB} /$ follower, respectively. The number of participants represents the number of endorsers who have retweeted the tweets in the given tasks. The number of followers represents the total follower number of all participated endorsers. The total/average/maximum engagements represent the number of engagements generated by the participants. The six products used in our experiment are: Heart Rate Meter (HRM), Fitness Tracker (FT), Buddha Statue (ST), Bracelet (BL), Ear Ring (ER), and Lapis Lazuli (LP). HRM and FT were sold by one vendor, and the other four products by another vendor. 
Detailed task-by-task participation and engagement statistics are shown in Table 3. There is a clear decline in the number of comments and retweets generated per task over time. The number of likes is also decreasing but not as fast. We stopped the experiment after week 8 due to this saturation effect. To make sure that the tasks did not run out of budget before they were closed for participation, we tried different budgets $(100,200$, and 300RMB) in the first four weeks and found that 200RMB was more than enough. Therefore, the budgets for all the tasks in the subsequent weeks are 200RMB. The distribution of engagement generated by participating endorsers is shown in Table 4. Most endorsements do not generate any engagement.

Table 4. Distribution of Engagements Generated by Individual Endorsers

\begin{tabular}{|l|lllllllllll|}
\hline Type & Distribution & 0 & 1 & 2 & 3 & 4 & $5 \sim 10$ & $>10$ & Mean & SD & Max \\
\hline likes & 2072 & 145 & 14 & 4 & 4 & 1 & 1 & 0.10 & 0.50 & 15 \\
comments & 2104 & 69 & 21 & 15 & 5 & 16 & 11 & 0.22 & 1.59 & 34 \\
retweets & 2130 & 50 & 9 & 15 & 7 & 15 & 15 & 0.23 & 1.71 & 36 \\
\hline
\end{tabular}

Table 5. Description of Independent Variables

\begin{tabular}{|ll|}
\hline $\begin{array}{l}\text { Variables } \\
\text { isEligible }\end{array}$ & Description \\
\hline $\begin{array}{l}\text { Incentive } \\
\text { payRate } \\
\text { actRwd } \\
\text { avgRwd } \\
\text { gain }\end{array}$ & Whether a endorser is eligible for a given task (for selection equation only) \\
loss & $\begin{array}{l}\text { Pay rate per follower (either 0.0002 or 0.0004 RMB/follower) } \\
\text { Actual reward upon approval, net of commission fee }\end{array}$ \\
\hline $\begin{array}{l}\text { Social Media Fan Base } \\
\text { followers } \\
\text { verifiedRatio }\end{array}$ & $\begin{array}{l}\text { Max(0, actRwd -avgRwd) } \\
\text { Max(0, avgRwd- actRwd) }\end{array}$ \\
\hline $\begin{array}{l}\text { Prior Activity Level } \\
\text { tweetNum } \\
\text { taskNum } \\
\text { approvalRate }\end{array}$ & $\begin{array}{l}\text { Number of followers on weibo.com } \\
\text { Percentage of verified followers in all followers }\end{array}$ \\
\hline $\begin{array}{l}\text { Community Embeddedness } \\
\text { regDays } \\
\text { friends }\end{array}$ & $\begin{array}{l}\text { Number of tweets posted on weibo.com } \\
\text { Total number of tasks participated in the past }\end{array}$ \\
\hline $\begin{array}{l}\text { Other } \\
\text { group } \\
\text { referralRwd } \\
\text { times }\end{array}$ & $\begin{array}{l}\text { Percentage of approved tasks in the past } \\
\text { Number of days an endorser has registered on weituitui.com (rescaled to [0,1]) }\end{array}$ \\
\hline
\end{tabular}

We collect data on the characteristics of endorsers by scraping their profiles on weituitui.com, which include their information on both weituitui.com and weibo.com. The information on weituitui.com includes the number of verified followers, the number of tasks participated in, the total amount of reward earned, the total referral income, the number of friends on weituitui's internal social network, and how long ago one registered on weituitui.com. The information on weibo.com includes the number of followers and the 
number of tweets (including retweets). In Table 5, we summarize the independent variables used for our analysis in six different categories. We focus on those variables that advertisers can set or observe and hence use for targeting. These variables fall into four categories: incentive, social media fan base, prior activity level, and community embeddedness. For incentive, in addition to pay rate, we also compute the actual reward an endorser will receive upon approval, which allows us to better account for the special pricing scheme showing in Figure 1. Note that the actual reward for ineligible participants will be zero regardless of their number of verified followers. ${ }^{7}$ As we discussed earlier, the effect of incentive may depend on some reference level. We choose the average reward per task in the past as the reference point and then derive the gain and loss for each endorser. The variables in the "Other" category, such as "referralRwd" and "times", are specific to the platform and our experimental design, and are used merely as controls. They are not of substantive interest.

Table 6. Key Statistics on Independent Variables

\begin{tabular}{|l|lllll|lllll|}
\hline \multirow{2}{*}{\multicolumn{1}{|c}{ Variables }} & \multicolumn{7}{c|}{ Entire Dataset } & \multicolumn{5}{c|}{ Subset of Participants } \\
\cline { 2 - 10 } & Mean & Median & Min & Max & SD & Mean & Median & Min & Max & SD \\
\hline isEligible & 0.25 & 0.00 & 0.00 & 1.00 & 0.43 & 0.96 & 1.00 & 0.00 & 1.00 & 0.20 \\
payRate & 2.97 & 2.00 & 2.00 & 4.00 & 1.00 & 2.98 & 2.00 & 2.00 & 4.00 & 1.00 \\
$\log$ (actRwd) & -5.53 & -6.91 & -6.91 & 5.37 & 2.44 & -1.14 & -1.05 & -6.91 & 4.29 & 1.54 \\
$\log$ (gain) & -6.51 & -6.91 & -6.91 & 5.27 & 1.30 & -4.68 & -6.91 & -6.91 & 3.69 & 2.71 \\
$\log (\operatorname{loss})$ & -6.18 & -6.91 & -6.91 & 3.27 & 1.94 & -4.44 & -6.91 & -6.91 & 2.74 & 2.71 \\
$\log ($ avgRwd) & -0.92 & -0.92 & -6.91 & 3.48 & 0.97 & -0.72 & -0.84 & -2.80 & 3.48 & 0.72 \\
$\log$ (followers) & 6.93 & 6.84 & 2.48 & 15.42 & 2.02 & 7.88 & 7.76 & 2.83 & 14.51 & 1.97 \\
verifiedRatio & 0.44 & 0.46 & 0.00 & 1.00 & 0.24 & 0.45 & 0.46 & 0.00 & 1.00 & 0.25 \\
$\log$ (tweetNum) & 5.90 & 5.99 & 0.00 & 11.26 & 1.75 & 6.57 & 6.66 & 0.00 & 11.26 & 1.61 \\
$\log$ (taskNum) & 2.48 & 2.30 & 0.00 & 8.70 & 1.87 & 4.54 & 4.76 & 0.00 & 8.70 & 1.81 \\
approvalRate & 0.74 & 0.82 & 0.00 & 1.00 & 0.28 & 0.83 & 0.87 & 0.00 & 1.00 & 0.16 \\
regDays & 0.21 & 0.18 & 0.00 & 1.00 & 0.15 & 0.21 & 0.17 & 0.00 & 1.00 & 0.19 \\
$\log$ (friends) & 0.38 & 0.00 & 0.00 & 2.77 & 0.71 & 0.60 & 0.00 & 0.00 & 2.77 & 0.90 \\
$\log$ (referralRwd) & -5.54 & -6.91 & -6.91 & 7.17 & 2.94 & -4.69 & -6.91 & -6.91 & 5.45 & 3.55 \\
times & 0.02 & 0.00 & 0.00 & 4.00 & 0.15 & 0.09 & 0.00 & 0.00 & 4.00 & 0.32 \\
\hline
\end{tabular}

Table 7. Correlation between Independent Variables

\begin{tabular}{|c|c|c|c|c|c|c|c|c|c|c|c|c|c|c|c|}
\hline & isEligible & $\begin{array}{l}\text { pay } \\
\text { Rate }\end{array}$ & $\log (\mathrm{actRwd})$ & $\log$ (gain) & $\log (\operatorname{loss})$ & $\log (\operatorname{avgRwd})$ & $\log$ (followers) & $\begin{array}{c}\text { verified } \\
\text { Ratio }\end{array}$ & $\log ($ tweetNum) & $\log ($ taskNum) & $\begin{array}{c}\text { approval } \\
\text { Rate }\end{array}$ & regDays & $\log$ (friends) & $\begin{array}{c}\log \text { (referral } \\
\text { Rwd) }\end{array}$ & times \\
\hline isEligible & 1.00 & & & & & & & & & & & & & & \\
\hline payRate & 0.00 & 1.00 & & & & & & & & & & & & & \\
\hline $\log (\mathrm{actRwd})$ & 0.98 & 0.01 & 1.00 & & & & & & & & & & & & \\
\hline $\log$ (gain) & 0.53 & 0.05 & 0.60 & 1.00 & & & & & & & & & & & \\
\hline $\log ($ loss $)$ & 0.65 & -0.02 & 0.60 & -0.11 & 1.00 & & & & & & & & & & \\
\hline $\log (\operatorname{avgRwd})$ & 0.00 & 0.00 & 0.04 & -0.10 & 0.21 & 1.00 & & & & & & & & & \\
\hline $\log$ (followers) & 0.00 & 0.00 & 0.08 & 0.16 & -0.07 & 0.36 & 1.00 & & & & & & & & \\
\hline verifiedRatio & 0.00 & 0.00 & 0.01 & 0.04 & -0.04 & -0.08 & -0.34 & 1.00 & & & & & & & \\
\hline $\log$ (tweetNum) & 0.00 & 0.00 & 0.04 & 0.07 & -0.03 & 0.17 & 0.49 & -0.28 & 1.00 & & & & & & \\
\hline $\log ($ taskNum $)$ & 0.00 & 0.00 & 0.02 & -0.04 & 0.13 & 0.29 & 0.21 & -0.04 & 0.22 & 1.00 & & & & & \\
\hline approvalRate & 0.00 & 0.00 & 0.01 & 0.07 & 0.06 & 0.00 & 0.11 & -0.02 & 0.10 & 0.21 & 1.00 & & & & \\
\hline regDays & 0.00 & 0.00 & 0.02 & 0.01 & 0.01 & 0.07 & 0.23 & -0.10 & 0.27 & 0.44 & 0.09 & 1.00 & & & \\
\hline $\log$ (friends) & 0.00 & 0.00 & 0.00 & -0.04 & 0.07 & 0.10 & 0.00 & -0.02 & 0.07 & 0.40 & 0.02 & 0.22 & 1.00 & & \\
\hline $\log ($ referralRwd $)$ & 0.00 & 0.00 & 0.00 & -0.03 & 0.07 & 0.14 & 0.07 & -0.04 & 0.10 & 0.42 & 0.09 & 0.29 & 0.36 & 1.00 & \\
\hline times & -0.04 & 0.00 & -0.04 & -0.02 & -0.03 & 0.03 & 0.07 & 0.00 & 0.06 & 0.15 & 0.05 & 0.00 & 0.04 & 0.04 & 1.00 \\
\hline
\end{tabular}

\footnotetext{
${ }^{7}$ We have also tried an alternative version of reward which does not distinguish between eligible and ineligible endorsers in computing expected rewards. That is, even ineligible endorsers can have non-zero rewards. In our later analyses, we find that this alternative coding produces very similar findings but worse model fit.
} 
The summary statistics of the independent variables are shown in Table 6. The characteristics of participating endorsers are clearly different from those of the whole population, which is evidence of selfselection. The correlations among the independent variables are shown in Table 7. Except for the expected correlations with variables representing incentive, the two manipulated variables "isEligible" and "payRate" have zero correlation with other variables, indicating effective randomization. isEligible is correlated with incentive because only eligible endorsers can have positive actual reward (see footnote 7).

\subsection{Model-Free Analysis of Manipulation Effects}

To provide some intuition regarding how the manipulations affect the participation and effectiveness of endorsers, we compare the participation rates and generated engagements between eligible vs. ineligible and between high-pay rate vs. low-pay rate endorsers. In addition, given that eligibility and pay rate may affect the effort level of endorsers, we also compare the effort levels of endorsers in different treatment groups. In paid retweeting campaigns similar to ours, the only place where endorsers can show differentiated efforts lies in the composition of the comment included in the retweet, if any. Two metrics that reflect the effort level of an endorser in composing a comment is the length of comment (namely the number of words ${ }^{8}$ ) and the use of emoji (yes or no). The former metric is commonly used to measure the effort level of respondents (Singer and Ye 2013).

Table 8. Effects of Manipulated Variables

\begin{tabular}{|c|c|c|c|c|c|c|c|}
\hline \multirow{2}{*}{\multicolumn{2}{|c|}{ Manipulated Variables }} & \multirow{3}{*}{$\begin{array}{l}\text { Participation rate } \\
3.78 \%\end{array}$} & \multicolumn{2}{|c|}{ Effort Levels in Retweets } & \multicolumn{3}{|c|}{ Engagements } \\
\hline & & & \# words attached & Use of emoji & Likes & Comments & Retweets \\
\hline \multirow{3}{*}{ Eligibility } & Eligible endorsers & & $16.54(11.28)$ & $9.77 \%$ & $0.10(0.51)$ & $0.22(1.57)$ & $0.22(1.70)$ \\
\hline & Ineligible endorsers & $0.05 \%$ & $15.34(12.09)$ & $5.49 \%$ & $0.09(0.28)$ & $0.33(1.89)$ & $0.42(1.96)$ \\
\hline & ANOVA test ( $p$-value) & $<0.001$ & 0.32 & 0.18 & 0.82 & 0.50 & 0.28 \\
\hline \multirow{3}{*}{ Pay Rate } & Low-payed endorsers & $0.97 \%$ & $16.59(11.01)$ & $8.11 \%$ & $0.10(0.44)$ & $0.28(2.02)$ & $0.27(2.12)$ \\
\hline & High-payed endorsers & $1.00 \%$ & $16.39(11.63)$ & $11.15 \%$ & $0.10(0.56)$ & $0.16(0.93)$ & $0.18(1.12)$ \\
\hline & ANOVA test ( $p$-value) & 0.61 & 0.68 & 0.01 & 0.98 & 0.08 & 0.19 \\
\hline
\end{tabular}

Reported values are means, with standard deviation in brackets. Both effort levels and engagements are conditional on participation.

Table 8 contrasts the average participation rates, effort levels, and engagements of eligible vs. ineligible (high-payed vs. low-payed) endorsers. The p-values from ANOVA test are provided. As expected, eligibility has a strong effect on participation but no effect on effort or engagement level. Therefore, eligibility is indeed a valid instrument, as expected. However, the effects of pay rate may be a bit surprising, as there are no significant differences between high and low pay rate except for the usage of emoji. The most likely explanation is that the payment for $77 \%$ of endorsers is not affected by high vs. low pay rates, due to weituitui's constraint on the reward structure, and that the effect of high vs. low pay rate on incentives received varies as a function of the number of verified followers for the remaining $23 \%$ (Figure 1).

\footnotetext{
${ }^{8}$ In Chinese, one word is represented by one character, so the number of characters is the same as the number of words.
} 


\section{Model}

The data analysis presents two challenges. First, engagement is observed only for those endorsers who participate in the task, and the effectiveness of participants may not be representative of the whole population. This is commonly known as the sample selection problem (Heckman 1979). Second, an endorser can participate in more than one task and the resulting observations on the same endorser may not be independent. While both the sample selection and repeated observation problems are common in the literature and can be addressed effectively when they appear separately, little has been done to address both problems jointly, especially when the dependent variable is counts. We propose a model to deal with both problems. We first present our approach to model participation and potential effectiveness of endorsers jointly in Section 5.1, and then discuss its connection to existing models in Section 5.2. We elaborate on how to compute some effects of substantive interest in Section 5.3.

\subsection{Sample Selection Model with Correlated Random Effects}

We model likes, comments, and retweets separately. For each of these outcomes, there are two equations in our model: the selection or participation equation captures what affects an endorser's participation, and the outcome equation captures what affects an endorser's potential effectiveness in generating engagement. The potential effectiveness is not conditional on participation, which allows us to gain insights on the entire population of endorsers, not only on those who participated. We use boldface letters to represent vectors and matrices. For notational compactness, we use row vectors throughout the paper.

Following the standard sample selection model (Greene 2009; Heckman 1979), we use a probit model for the selection equation. Letting the variable $z_{i t}$ indicate whether endorser $i$ participates in task $t$, the participation decision is given by

$$
z_{i t}=\mathbf{1}\left(\boldsymbol{\alpha} \boldsymbol{w}_{i t}^{\prime}+\delta u_{i}+\xi_{i t}>0\right)
$$

where $\boldsymbol{w}_{i t}$ includes an intercept and the sets of variables that affects the participation decision of endorser $i$ in task $t$. The variables in $\boldsymbol{w}_{i t}$ include characteristics of endorser $i$, characteristics of task $t$, the characteristics specific to the endorser-task dyad, and the exclusion variable (see Table 5). They also include 15 dummy variables for each pair of identical tasks posted that vary only on pay rate (the intercept captures the sixteenth pair). These dummies absorb any task-specific effect apart from pay rate, including characteristics of the product featured, characteristics of our post on weituitui, and temporal shocks. Note that the two paired tasks share the same fixed effect as they are identical except for pay rate which is controlled separately. The random terms $u_{i} \sim N(0,1)$ and $\xi_{i t} \sim N(0,1)$ capture endorser and endorser-task level unobserved characteristics that affect the participation decision, respectively. The selection equation given above is a probit model with random effects (Butler and Moffitt 1982). 
Since the engagements (including likes, comments, and retweets) are all counts, and since the data feature both overdispersion (see Table 4) and repeated observations, we use a Poisson lognormal model with random effects for the outcome equation. Let $y_{i t}^{*}$ be the potential outcome (i.e., the number of likes, comments, or retweets) of endorser $i$ on task $t$. The outcome equation is given by

$$
E\left[y_{i t}^{*} \mid \boldsymbol{x}_{i t}, \varepsilon_{i}, \epsilon_{i t}\right]=\lambda_{i t}=\exp \left(\boldsymbol{\beta} \boldsymbol{x}_{i t}^{\prime}+\sigma \varepsilon_{i}+\gamma \epsilon_{i t}\right)
$$

where $\boldsymbol{x}_{i t}$ includes an intercept and the set of variables that affects the potential engagement generated by endorser $i$ for task $t$. The only difference between $\boldsymbol{x}_{i t}$ and $\boldsymbol{w}_{i t}$ is that only $\boldsymbol{w}_{i t}$ includes the exclusion variable. Our outcome equation accounts for two levels of heterogeneity: $\varepsilon_{i} \sim N(0,1)$ and $\epsilon_{i t} \sim N(0,1)$ capture the effect of endorser and endorser-task level unobserved characteristics, respectively. When $\sigma=$ 0, the above model simplifies to the Poisson lognormal model (Greene 2009), which often yields similar estimates to the Negative Binomial model. In our data, we find that the above model with two levels of heterogeneity fit the data substantially better than the zero-inflated Poisson and the Negative Binomial models.

The error terms in the selection and outcome equations need not be independent. Specifically, the endorser-level unobserved characteristics that affect selection or participation may also affect outcomes, and so may endorser-task level unobserved characteristics. As a result, we further assume that the endorser and endorser-task level error terms are bivariate normally distributed, with a correlation of $\rho$ and $\tau$, respectively.

$$
\left(\begin{array}{c}
u_{i} \\
\varepsilon_{i}
\end{array}\right) \sim N\left(\left(\begin{array}{l}
0 \\
0
\end{array}\right),\left(\begin{array}{ll}
1 & \rho \\
\rho & 1
\end{array}\right)\right),\left(\begin{array}{l}
\xi_{i t} \\
\epsilon_{i t}
\end{array}\right) \sim N\left(\left(\begin{array}{l}
0 \\
0
\end{array}\right),\left(\begin{array}{ll}
1 & \tau \\
\tau & 1
\end{array}\right)\right)
$$

Compared to the existing sample selection models (Greene 2009; Heckman 1979; Winkelmann 1998), our model not only takes into account random effects, but also allows the random effects to be correlated. Letting $T_{i}$ be the number of tasks endorser $i$ can potentially participate, the likelihood of all observations on endorser $i$ can be written as

$$
\begin{gathered}
L_{i}=P\left(y_{i 1}^{*}, \ldots, y_{i T_{i}}^{*} ; z_{i 1}, \ldots, z_{i T_{i}} \mid \boldsymbol{x}_{i 1}, \ldots, \boldsymbol{x}_{i T_{i}}, \boldsymbol{w}_{i 1}, \ldots, \boldsymbol{w}_{i T_{i}}\right) \\
=\int_{-\infty}^{\infty} \phi\left(\varepsilon_{i}\right) d \varepsilon_{i} \int_{-\infty}^{\infty} f\left(u_{i} \mid \varepsilon_{i}\right) d u_{i} \prod_{t=1}^{T_{i}} \int_{-\infty}^{\infty} P\left(y_{i t}^{*} \mid \boldsymbol{x}_{i t}, \varepsilon_{i}, \epsilon_{i t}\right)^{z_{i t}} P\left(z_{i t} \mid \boldsymbol{w}_{i t}, u_{i}, \epsilon_{i t}\right) \phi\left(\epsilon_{i t}\right) d \epsilon_{i t}
\end{gathered}
$$

where $P\left(y_{i t}^{*} \mid x_{i t}, \varepsilon_{i}, \epsilon_{i t}\right)=\frac{\lambda_{i t} y_{i t} e^{-\lambda_{i t}}}{y_{i t} !}$, as given by the conditional Poisson distribution. In the likelihood function, $P\left(y_{i t}^{*} \mid x_{i t}, \varepsilon_{i}, \epsilon_{i t}\right)$ only factors in when $z_{i t}=1$, as $y_{i t}^{*}$ is only observed for participating endorsers. The conditional density $f\left(u_{i} \mid \varepsilon_{i}\right)$ is derived from the bivariate normal distribution. The likelihood of our model does not have a closed-form representation. However, it can be numerically approximated by the 
Gauss-Hermite quadrature method (Abramowitz and Stegun 1972; Greene 2009). Appendix A provides more details on the likelihood derivation and parameter estimation. Our model can deal with outcomes following other distributions by changing the distributional assumption on $P\left(y_{i t}^{*} \mid x_{i t}, \varepsilon_{i}, \epsilon_{i t}\right)$.

The random terms for different types of engagement may be correlated. However, since the three types of engagements have exactly the same set of regressors in our analysis, estimating the equations for different types of engagements independently, as if there are no correlation across engagements, will give identical estimates (Kruskal 1968).

\subsection{Connections with Existing Models}

To convey the connection of the proposed model with existing models, Table 9 summarizes potential nested models based on the specification of endorser and endorser-task level random terms. For both endorser and endorser-task random terms, Table 9 considers three possibilities: the random term is not specified in either the selection or outcome equation, it is specified in both equations but not correlated, and it is specified in both equations and correlated. For simplicity, Table 9 ignores cases in which the random term is specified in one equation but not the other. To allow for unrestricted correlation between the random terms across equations, all the terms are assumed to be normally distributed. In count models, normal error (as in the Poisson Lognormal model) often yields very similar results with Exponential-Gamma error (as in the Negative Binomial model). The models in Table 9 are named based on the outcome equation and the correlation(s). The selection equation is probit or probit with random effects by default. Existing models for panel count data with sample selection are rather restricted. The proposed model nests them.

Table 9. Models for Panel Count Data with Sample Selection

\begin{tabular}{|c|c|c|c|}
\hline Endorser level $\left(\varepsilon_{i}\right) \quad$ Endorder-task level $\left(\epsilon_{i t}\right)$ & No & Uncorrelated & Correlated \\
\hline No & Poisson & PLN (Greene 2009) & PLN with SS (Greene 2009) \\
\hline Uncorrelated & Poisson RE (Hall 2000) & & \\
\hline Correlated & & & PLN with SS and CRE (Proposed) \\
\hline
\end{tabular}

* PLN: Poisson Lognormal model; RE: random effects; SS: sample selection, namely with correlation on endorser-task level random terms; CRE: correlated random effects, namely with correlation on endorser level random terms. Empty cells represent models that are missing in the literature.

\subsection{Relative Partial Effects on Potential and Actual Outcome}

One question of interest to marketers is how much the mean potential outcome $E\left[\boldsymbol{y}_{i t}^{*} \mid \boldsymbol{x}_{i t}\right]$ varies with respect to the changes in endorsers' characteristics $\boldsymbol{x}_{i t}$. Integrating out $\varepsilon_{i}$ and $\epsilon_{i t}$ in equation (2) yields

$$
E\left[\boldsymbol{y}_{i t}^{*} \mid \boldsymbol{x}_{i t}\right]=E_{\varepsilon_{i}} E_{\epsilon_{i t}}\left[E\left[\boldsymbol{y}_{i t}^{*} \mid \boldsymbol{x}_{i t}, \varepsilon_{i}, \epsilon_{i t}\right]\right]=\exp \left(\boldsymbol{\beta} \boldsymbol{x}_{i t}^{\prime}+\frac{\sigma^{2}+\gamma^{2}}{2}\right)
$$

Therefore, the relative partial effects of $\boldsymbol{x}_{i t}$ on the mean potential outcome is simply $\frac{\partial \log E\left[\boldsymbol{y}_{i t}^{*} \mid \boldsymbol{x}_{i t}\right]}{\partial \boldsymbol{x}_{i t}}=$ $\boldsymbol{\beta}$. The absolute partial effects of $\boldsymbol{x}_{i t}$ on $E\left[\boldsymbol{y}_{i t}^{*} \mid \boldsymbol{x}_{i t}\right]$ is $\exp \left(\boldsymbol{\beta} \boldsymbol{x}_{i t}^{\prime}+\frac{\sigma^{2}+\gamma^{2}}{2}\right) \boldsymbol{\beta}$. We focus on the relative 
partial effects as the absolute partial effects are quite sensitive to outliers in the data due to the exponential term.

Advertisers are interested in how the characteristics of endorsers impact not only the potential outcome but also the actual or observable outcome. If an endorser chooses not to participate, the engagements generated would be zero. Therefore, the relationship between the actual outcome $y_{i t}$ and the potential outcome $y_{i t}^{*}$ can be written as

$$
y_{i t}=z_{i t} y_{i t}^{*}
$$

Given that $z_{i t}$ and $y_{i t}^{*}$ are independent conditional on $\boldsymbol{x}_{i t}, \boldsymbol{w}_{i t}, u_{i}, \varepsilon_{i}, \epsilon_{i t}$, the conditional mean outcome can be written as

$$
E\left[y_{i t} \mid \boldsymbol{x}_{i t}, \boldsymbol{w}_{i t}, u_{i}, \varepsilon_{i}, \epsilon_{i t}\right]=P\left(z_{i t}=1 \mid \boldsymbol{w}_{i t}, u_{i}, \epsilon_{i t}\right) E\left[y_{i t}^{*} \mid \boldsymbol{x}_{i t}, \varepsilon_{i}, \epsilon_{i t}\right]
$$

The unconditional (i.e., not conditional on any unobserved variable) mean outcome can be obtained by integrating out $u_{i}, \varepsilon_{i}$ and $\epsilon_{i t}$ in equation (6) (see Appendix B for details).

$$
E\left[y_{i t} \mid \boldsymbol{x}_{i t}, \boldsymbol{w}_{i t}\right]=E_{u_{i}} E_{\varepsilon_{i} \mid u_{i}} E_{\epsilon_{i t}}\left[E\left[y_{i t} \mid \boldsymbol{x}_{i t}, \boldsymbol{w}_{i t}, u_{i}, \varepsilon_{i}, \epsilon_{i t}\right]\right]
$$

The relative partial effect of a variable $s_{i t}$ on the mean outcome can be written as

$$
g_{s_{i t}}=\frac{\partial \log E\left[\boldsymbol{y}_{i t} \mid \boldsymbol{x}_{i t}, \boldsymbol{w}_{i t}\right]}{\partial s_{i t}}=c_{i t} \alpha_{s}+\beta_{s}
$$

where $\alpha_{s}$ represents the corresponding coefficient in $\boldsymbol{\alpha}$ if $s_{i t}$ belongs to $\boldsymbol{w}_{i t}$, otherwise 0. Similarly, $\beta_{s}$ represents the corresponding coefficient in $\boldsymbol{\beta}$ if $s_{i t}$ belongs to $\boldsymbol{x}_{i t}$, otherwise 0 . The functional form of coefficient $c_{i t}$, which is always positive, is given in Appendix C. The standard errors of the relative partial effects can be estimated using the delta method (see Appendix C). It can be seen from equation (8) that, if a variable only affects the participation equation, then its directional effect on actual outcome is consistent with its directional effect on participation. If a variable only affects the outcome equation, then its impact on potential and actual outcomes are in the same direction. However, for variables appearing in both participation and outcome equations, their effects on potential and actual outcomes might have opposite signs.

\section{Results}

\subsection{Selection of Incentive Variables}

The incentive of endorsers can be represented in three different ways. The first is to use the pay rate which are exogenously manipulated. However, due to the policies of weituitui.com, $77 \%$ of endorsers are indifferent between the two pay rates. The second way is to use the actual reward upon approval, which can account for institutional details. The third is to separate rewards into gains and losses, as posited by prospect theory. An additional consideration is that rewards, gains and losses may have constant or decreasing returns to scale, the latter of which can be accounted for by a log transformation (Hardie et al 1993). 
Rather than assuming a priori one of these representations, we choose the one that best fits the data. Since the incentive of endorsers is not contingent on performance, we expect little impact of incentive on outcome. Therefore, we base our choice of representation on how well each fits the participation data. Table 10 reports the model fit of different incentive representations. The logged gain-loss representation fits the data best on all three model fitness metrics, as expected from prospect theory. Therefore, in our following analysis, we represent incentive in a gain-loss framework with log transformation. Note, the logged gainloss representation fits the data best also if we take outcomes into consideration.

Table 10. Selection of Incentive Variables

\begin{tabular}{|l|l|ll|ll|}
\hline & & \multicolumn{2}{|c|}{ Linear } & \multicolumn{2}{c|}{ Log } \\
\hline & PayRate & Reward & GainLoss & Reward & GainLoss \\
$\mathbf{- 2 * L L}$ & 13315.9 & 13313.0 & 13289.0 & 13269.3 & 13239.4 \\
AIC & 13371.9 & 13369.0 & 13349.0 & 13325.3 & 13299.4 \\
BIC & 13661.3 & 13658.4 & 13659.0 & 13614.7 & 13609.5 \\
\hline
\end{tabular}

\subsection{Main Results}

Table 11 reports the parameter estimates using our main model in Section 5. To ease comparison, the estimates for the participation equations for each of the three outcomes are presented side by side, followed by the estimates for the three outcome equations. All the heterogeneity and correlation parameters, as well as model fitness metrics, are shown in the outcome column. We discuss our findings on the independent variables category by category.

Incentive. The effect of losses is greater than that of gains, which is consistent with previous evidence of loss aversion (Hardie et al. 1993; Kalyanaram and Winer 1995). Gains have no significant effect on participation, which is consistent with previous evidence that additional incentives do not impact performance when workers already feel adequately remunerated (Cohn et al. 2015). Our findings suggest that improving participation rates through increasing incentive can be inefficient. To improve participation rates of paid endorsement campaigns, it may be better to provide an incentive just comparable to endorsers' past rewards and then focus on providing non-monetary incentives. The finding that neither gains nor losses have an effect on outcome is consistent with previous findings that incentives need not affect performance unless they are contingent on performance (Singer and Ye 2013).

We also estimate a set of models using the exogenously manipulated pay rate to represent incentive (Table OA.1 in the Online Appendix). We find that pay rate has no effect on participation, even though the higher pay rate used in our experiment exceeds or equals to $96 \%$ of linear prices ever used on weituitui. This is consistent with the model-free evidence reported in Section 4.2. ${ }^{9}$ Using pay rate instead of gains and

\footnotetext{
${ }^{9}$ Additional analysis shows that pay rate has no effect on participation even for endorsers whose incentives are sensitive to pay rate. We conducted this analysis by interacting the pay rate with a dummy variable indicating whether an endorser is sensitive to pay rate, i.e., whether the endorser has more than 1250 verified followers. Neither the pay rate nor the interaction term have a significant effect.
} 
losses does not affect the coefficients of other variables much. Using other types of incentive representations listed in Table 10 yields similar findings (Tables OA.1-4 in the Online Appendix). In the rest of our discussion, we focus on the results reported in Table 11 which give the best model fits.

Table 11. Parameter Estimates for Different Types of Engagements (Log GainLoss)

\begin{tabular}{|c|c|c|c|c|c|c|}
\hline & \multicolumn{3}{|c|}{ Selection } & \multicolumn{3}{|c|}{ Outcome } \\
\hline & likes & comments & retweets & likes & comments & retweets \\
\hline $\begin{array}{l}\text { Exclusion Variable } \\
\text { isEligible }\end{array}$ & $2.724 * * *$ & $2.723^{* * *}$ & $2.720 * * *$ & & & \\
\hline $\begin{array}{l}\text { Incentive } \\
\qquad \begin{array}{l}\log \text { (gain) } \\
\log (\text { loss }) \\
\log (\text { avgRwd })\end{array}\end{array}$ & $\begin{array}{l}0.007 \\
-0.073^{* * *} \\
0.034\end{array}$ & $\begin{array}{l}0.008 \\
-0.074 * * * \\
0.036\end{array}$ & $\begin{array}{l}0.009 \\
-0.073^{* * *} \\
0.037\end{array}$ & $\begin{array}{l}-0.039 \\
0.022 \\
-0.092\end{array}$ & $\begin{array}{l}-0.011 \\
0.146 \\
0.174\end{array}$ & $\begin{array}{l}-0.061 \\
0.095 \\
0.333\end{array}$ \\
\hline $\begin{array}{c}\text { Social Media Fanbase } \\
\log \text { (followers) } \\
\text { verifiedRatio }\end{array}$ & $\begin{array}{l}0.083^{* * * *} \\
0.256^{*}\end{array}$ & $\begin{array}{l}0.077 * * * \\
0.297 * *\end{array}$ & $\begin{array}{l}0.079 * * * \\
0.283 * *\end{array}$ & $\begin{array}{l}0.221 \\
0.652\end{array}$ & $\begin{array}{l}-0.039 \\
1.105\end{array}$ & $\begin{array}{l}-0.195 \\
0.989\end{array}$ \\
\hline $\begin{array}{c}\text { Prior Activity Level } \\
\log \text { (tweetNum) } \\
\log (\text { taskNum }) \\
\text { approvalRate }\end{array}$ & $\begin{array}{l}0.035 . \\
0.555^{* * * *} \\
0.063\end{array}$ & $\begin{array}{l}0.045^{*} \\
0.564^{* * *} \\
0.051\end{array}$ & $\begin{array}{l}0.039 \\
0.561 * * * \\
0.063\end{array}$ & $\begin{array}{l}0.001 \\
-0.379 * * * \\
0.704\end{array}$ & $\begin{array}{l}-0.478^{* * * *} \\
-0.483^{* * *} \\
1.708\end{array}$ & $\begin{array}{l}-0.252 * \\
-0.570 * * * \\
-0.300\end{array}$ \\
\hline $\begin{array}{l}\text { Community Embeddedness } \\
\text { regDays } \\
\log (\text { friends }) \\
\end{array}$ & $\begin{array}{l}-4.375^{* * *} \\
-0.088^{*} \\
\end{array}$ & $\begin{array}{l}-4.410 * * * \\
-0.084 *\end{array}$ & $\begin{array}{l}-4.358 * * * \\
-0.086^{*}\end{array}$ & $\begin{array}{l}0.102 \\
0.420^{* *}\end{array}$ & $\begin{array}{l}2.826^{*} \\
0.131 \\
\end{array}$ & $\begin{array}{l}3.258^{* *} \\
0.368 \\
\end{array}$ \\
\hline $\begin{array}{l}\text { Others } \\
\quad \log (\text { referralRwd) } \\
\text { times }\end{array}$ & $\begin{array}{l}-0.015 \\
-0.142 * *\end{array}$ & $\begin{array}{l}-0.017 \\
-0.142 * *\end{array}$ & $\begin{array}{l}-0.017 \\
-0.147 * *\end{array}$ & $\begin{array}{l}0.023 \\
-0.285 \\
\end{array}$ & $\begin{array}{l}0.003 \\
-0.102 \\
\end{array}$ & $\begin{array}{l}-0.095 \\
0.017 \\
\end{array}$ \\
\hline $\begin{array}{r}\text { Heterogeneity } \\
\delta \text { (selection) } \\
\sigma \text { (outcome) } \\
\gamma \text { (outcome) } \\
\end{array}$ & & & & $\begin{array}{l}1.310^{* * *} \\
1.609 * * * \\
0.097 \\
\end{array}$ & $\begin{array}{l}1.315^{* * * *} \\
2.190^{* * * *} \\
1.131^{* * *} \\
\end{array}$ & $\begin{array}{l}1.314 * * * \\
2.693 * * * \\
1.371 * * \\
\end{array}$ \\
\hline $\begin{array}{l}\text { Correlation } \\
\quad \rho \text { (endorser) } \\
\tau \text { (endorser-task) }\end{array}$ & & & & $\begin{array}{l}-0.216^{* * * *} \\
0.008\end{array}$ & $\begin{array}{l}-0.253^{* * *} \\
0.199\end{array}$ & $\begin{array}{l}-0.247 \text { *** } \\
0.312 \text {. }\end{array}$ \\
\hline $\begin{array}{l}\text { Fitness } \\
\text { Log Likelihood } \\
\text { AIC } \\
\text { BIC } \\
\end{array}$ & & & & $\begin{array}{l}-7229.7 \\
14583.4 \\
15224.2 \\
\end{array}$ & $\begin{array}{l}-7304.5 \\
14733.0 \\
15373.8 \\
\end{array}$ & $\begin{array}{l}-7216.5 \\
14557.0 \\
15197.8\end{array}$ \\
\hline
\end{tabular}

* Significance codes: ".” for $\mathrm{p}<10 \%$, “*” for $\mathrm{p}<0.05$, “**” for $\mathrm{p}<0.01$, and “***” for $\mathrm{p}<0.001$. For compactness, the intercept and the coefficients on the dummy variable "taskDummy" are omitted. The level of efforts required for an engagement: like $<$ comment $<$ retweet.

Social media fan base. Endorsers with more followers and a higher verified ratio are more likely to participate. One very plausible explanation is that endorsers with a greater number of verified followers derive greater status enhancement from relaying attractive deals than endorsers with fewer verified followers (Toubia and Stephen 2013) An alternative explanation is that, since followers and verified ratio determine the number of verified followers and hence affect the reward of endorsers, this finding indicates that those who are paid more are more likely to participate. This alternative explanation, however, is at odds with the finding that gains have no effect on participation.

Turning our attention to the outcome equation, we see that the number of followers has a marginally significant effect on likes, but not on comments or retweets. This finding, though a bit weak (but rather robust in our analyses), is consistent with our conjecture that the effect of followers may be smaller for 
forms of engagement that are more effortful. The reason is that higher levels of engagement are facilitated by strong ties, whereas the tie strength between endorsers and their fans decreases with the number of followers (Burke 2011; Katona et al. 2011; Roberts et al. 2009). This finding is consistent with previous findings that network size has a positive effect on overall influence in low-effort behaviors (e.g., (Yoganarasimhan 2012).

Prior activity level. Endorsers who tweeted more on microblogs and who participated in more tasks in the past are more likely to participate in our tasks, yet are less effective in generating engagements. This finding confirms our earlier conjecture that endorsers who are less selective tend to be less effective. Specifically, posting irrelevant or unsound content (e.g., ads) too often can hurt users' reputation in online communities (Barasch and Berger 2014; Bock et al. 2005), and hence their effectiveness.

The variable "approvalRate", defined as the percentage of approved tasks in the past, is a metric of endorsers' diligence. To our surprise, we find no significant effect of approval rate in the outcome equation, except for a marginally significant effect on comments. This suggests that approval rate might not be an ideal indicator of quality. Endorsers with higher approval rate might just be more skillful in fulfilling the requirements of advertisers. Given that approval rate has been widely used as a metric to judge the quality of workers in crowdsourcing services such as Amazon Mechanical Turk (Ipeirotis et al. 2010; Paolacci et al. 2010), this finding suggests that the construct validity of approval rate as a metric of quality may need more thorough investigation, at least in the context of paid endorsement campaigns.

Community embeddedness. Endorsers who have registered for a longer time and who have more friends on weituitui's internal social network are less likely to participate in a task, but more likely to generate certain types of engagements (comments and retweets for the number of days since registration, and likes for the number of friends). The opposite effects of these two variables on participation vs. effectiveness suggests that endorsers who are more embedded and respected within the community tend to be more selective, rendering them more effective in generating engagements.

Unobserved endorser traits. The negative correlation $\rho$ is of particular note. It indicates that the opposite effects on participation vs. effectiveness extend to unobserved endorser characteristics.

Engagement types. Among the three types of engagements, "likes" require the least effort as they do not involve any typing, and "retweet" require the most effort as they involve both commenting and sharing. The same is true for the three types of engagements on Facebook: likes, comments and shares (similar to retweets). Facebook assigns the largest weight to shares and the least weight to likes in their EdgeRank algorithm ${ }^{10}$.

\footnotetext{
${ }^{10} \mathrm{http} / / / \mathrm{www}$. socialbakers.com/blog/1304-understanding-increasing-facebook-edgerank
} 
The effects of variables in the outcome equations are often different for relatively high-effort engagement through comments and retweets versus low-effort engagement through likes. For example, it's harder for endorsers who tweet and endorse a lot to generate high-effort engagement (i.e., comments and retweets) than low-effort engagement (i.e., likes). The reverse is true for having been registered for a long time. These findings suggest that having been selective in the past and being a long-time endorser are associated with being more effective, not just in general but especially so for high-quality engagement.

\subsection{Robustness}

6.3.1 Identification. The validity of the exclusion restriction is critical to our analysis. One conceivable concern might be that, though the eligibility constraint was assigned randomly and independently of any endorser trait, the imposed (in)eligibility changed the endorsement behavior (e.g., effort level) of the endorser and hence affected her effectiveness indirectly. For example, ineligible endorsers may exert stronger effort than eligible endorsers in order to be approved, or exert lower effort given that they have lower faith in actually getting paid. If that is true, eligibility might not be truly exclusive. However, simple ANOVA analyses reported in Table 8 shows that eligibility has no effect on effort. More sophisticated multivariate analyses show that this conclusion is robust to controlling for the independent variables entering the outcome equation (Table OA.5 in the Online Appendix). The concern that eligibility might have affected the effort level and hence the effectiveness is not supported by the data.

Another concern about the validity of our analysis and findings is that the tasks may have interfered with each other, even though we used a unique tweet for each task. A first cause for concern is that there might be overlap between two endorsers' set of followers. If a follower is exposed to more than one endorser's retweets, there might be an attribution problem on the engagement by that follower. This type of interference is unlikely in our data since, unlike Twitter, Weibo allows users to engage (like, comment, retweet) on different retweets of the same tweet separately. If needed, the follower can like/comment/retweet all the retweets from different endorsers. Moreover, even if the follower decided to interact with only one of the retweets on the same product, the retweet the follower actually engages on is likely to be from the endorser who has the primary effect on the follower's decision.

A second cause of concern about interference is that, as we posted multiple tasks on the same product over time, there may have been a saturation effect if a follower saw the same product endorsed multiple times. While such interference may indeed have depressed the average effectiveness, there is no compelling reason to believe it would bias our estimates in the selection and outcome equations in opposite directions in a systematic manner. In other words, our main findings that participation and effectiveness are often at odds is not likely to be driven by the saturation effect. Moreover, the task level dummy variable and the control variable "times" already accounted for any main effect of task-level and endorser-level saturation on effectiveness. 
A final concern is that, among all participating endorsers, 16 participated in two tasks in a same group (i.e., two identical tasks on the same product at different pay rates), leading to a potential attribution problem between the two retweets on the same product retweeted by the same endorser. However, in the corresponding 16 (endorsers) *2 (tasks) *3 (types of engagements) observations, only 6 have non-zero engagements ( $\max$ is 3 and median is 1 ). The attribution issues on such a small number of observations with such low engagements are unlikely to bias our estimates substantially.

6.3.2 Robustness to Model Complexity. In Table 11, $\gamma$ is insignificant for likes and $\tau$ is insignificant for likes and comments (the significance for retweets is also only marginal), which might be a signal for over-specification. To examine whether our findings are an artifact of over-specification, we force $\tau$ (and $\gamma)$ to zero and re-estimate the parameters. The results in these simplified models are very similar to the full model (see Tables OA.6 in online Appendix). Since we are ignorant a priori about which of the parameters are significant, for the sake of full information disclosure, we report the results from the full model as main results. We further tried some additional simplified models, including removing random effects, removing dyadic heterogeneity, and removing all correlations (i.e., estimating selection and outcome equation models separately), and find that the findings of substantive interest are highly robust.

6.3.3 Robustness to Outliers. Among the 31 tasks, task 2 seems to be an outlier, with only 3 valid participations. This is because an endorser with half a million verified followers participated in this task soon after the task was posted and exhausted the budget of the task, which prevented other endorsers from participating. In fact, the other three tasks in the first week may also suffer somehow from this problem, due to the relatively lower budget. However, for tasks after the first week, this should not be an issue, as the budget was either double or triple that in week 1 . To assess whether our findings are driven by the potential outlier task(s) in week 1, we repeated our analysis first after removing task 2 and then after removing tasks 1-4 (all tasks in week 1). The estimates and findings of substantive interest are robust, except that the confidence bounds widen somewhat due to the smaller number of observations (Table OA.7 in Online Appendix).

\section{Implications for Program Design}

\subsection{Influencing Endorsers by Redesigning Tasks}

The results in Table 11 show that endorsers who are responsive to the campaigns are often less effective, whereas effective endorsers are often less responsive. To better understand the tension between responsiveness and effectiveness, we grouped endorsers into four cells in Table 12 based on their predicted responsiveness and effectiveness (Appendix D presents expressions for predicted values). Table 12 labels an endorser effective (responsive) if her predicted potential to generate engagements (predicted probability to participate) is above the mean of the data set. The percentages are first computed for individual tasks and 
then averaged over all tasks. While making predictions, we assume that every endorser is eligible for every task and the incentive for an endorser is her average reward per task in the past. This rules out the effects of the manipulated eligibility and incentive, allowing us to focus on the effects of endorsers' characteristics. The results are very similar if we assume that every endorser is paid at either the lower or higher rate.

Table 12. Distribution of Endorsers

\begin{tabular}{|l|ll|ll|ll|}
\hline \multirow{2}{*}{} & \multicolumn{2}{|c|}{ Likes } & \multicolumn{2}{c|}{ Comments } & \multicolumn{2}{c|}{ Retweets } \\
\cline { 2 - 7 } & Effective & Ineffective & Effective & Ineffective & Effective & Ineffective \\
\hline Responsive & $3.5 \%$ & $26.7 \%$ & $1.1 \%$ & $29.0 \%$ & $0.5 \%$ & $29.7 \%$ \\
Unresponsive & $32.6 \%$ & $37.2 \%$ & $25.6 \%$ & $44.3 \%$ & $30.2 \%$ & $39.6 \%$ \\
\hline
\end{tabular}

Only a very small percentage of endorsers are both effective and responsive. This is especially so for higher-effort engagements, i.e., comments and retweets. To improve the effectiveness of paid endorsement campaigns, advertisers may want to find ways to attract endorsers who are effective but unresponsive (e.g., endorsers who have registered for a long time and have many friends on weituitui.com). For example, to attract selective and effective endorsers, advertisers may want to experiment with designing ads that are less likely to hurt an endorser's reputation (e.g., native ads that look like organic tweets). They may also want to experiment with lowering the task requirements and offering tasks exclusively to endorsers who have registered for a long time and who have many friends on weituitui. This can be implemented by the written eligibility restrictions in the tasks.

In addition, advertisers may want to seek ways to improve the impact of responsive but ineffective endorsers (e.g., endorsers who tweet and endorse a lot). For example, if the expected participants are mostly those who are responsive but not effective, advertisers can increase the effort-related requirements in the tasks such as the minimal number of words and emojis in retweets, and the minimal number of people to be mentioned while retweeting.

In Online Appendix C, we further show that the four different types of endorsers in Table 12 tend to have different characteristics. Therefore, in practice, it's possible to "attract" effective but unresponsive endorsers and "enforce" responsive but ineffective endorsers at the same time by offering them different versions of tasks exclusively. For instance, if the objective of advertisers is to generate comments or retweets, advertisers can offer an "attract" task to endorsers who post few tweets and participate in few tasks, and an "enforce" task to endorsers who post a lot and participate a lot (see Online Appendix C).

\subsection{Boosting Potential vs. Actual Engagements by Targeting}

The tension between responsiveness and effectiveness further invites analysis of the relative partial effects of the independent variables on the actual vs. potential engagements. For simplicity, we call effects on the actual outcomes the "total effects" through both participation and potential outcomes. To generate a 
large number of engagements, an endorser needs to not only have high a potential to generate engagements, but also to actually participate in the campaign. The total effect of a variable on actual engagement can be computed using equation (8), which represents the percentage change of the engagements w.r.t. a unit change in the independent variable. Table 13 summarizes the total effects of the independent variables on actual engagements, which is first computed for each endorser-task dyad and then averaged over the entire population. The relative partial effects of independent variables on potential outcome are taken directly from Table 11, as we have shown in section 4.2 that $\frac{\partial \log E\left[\boldsymbol{y}_{i t}^{*} \mid \boldsymbol{x}_{i t}\right]}{\partial \boldsymbol{x}_{i t}}=\boldsymbol{\beta}$. The partial effects of independent variables on participation in Table 11 are also included in Table 13 to ease comparison.

Table 13. (Relative) Partial Effects on Participation, Potential and Actual Effectiveness

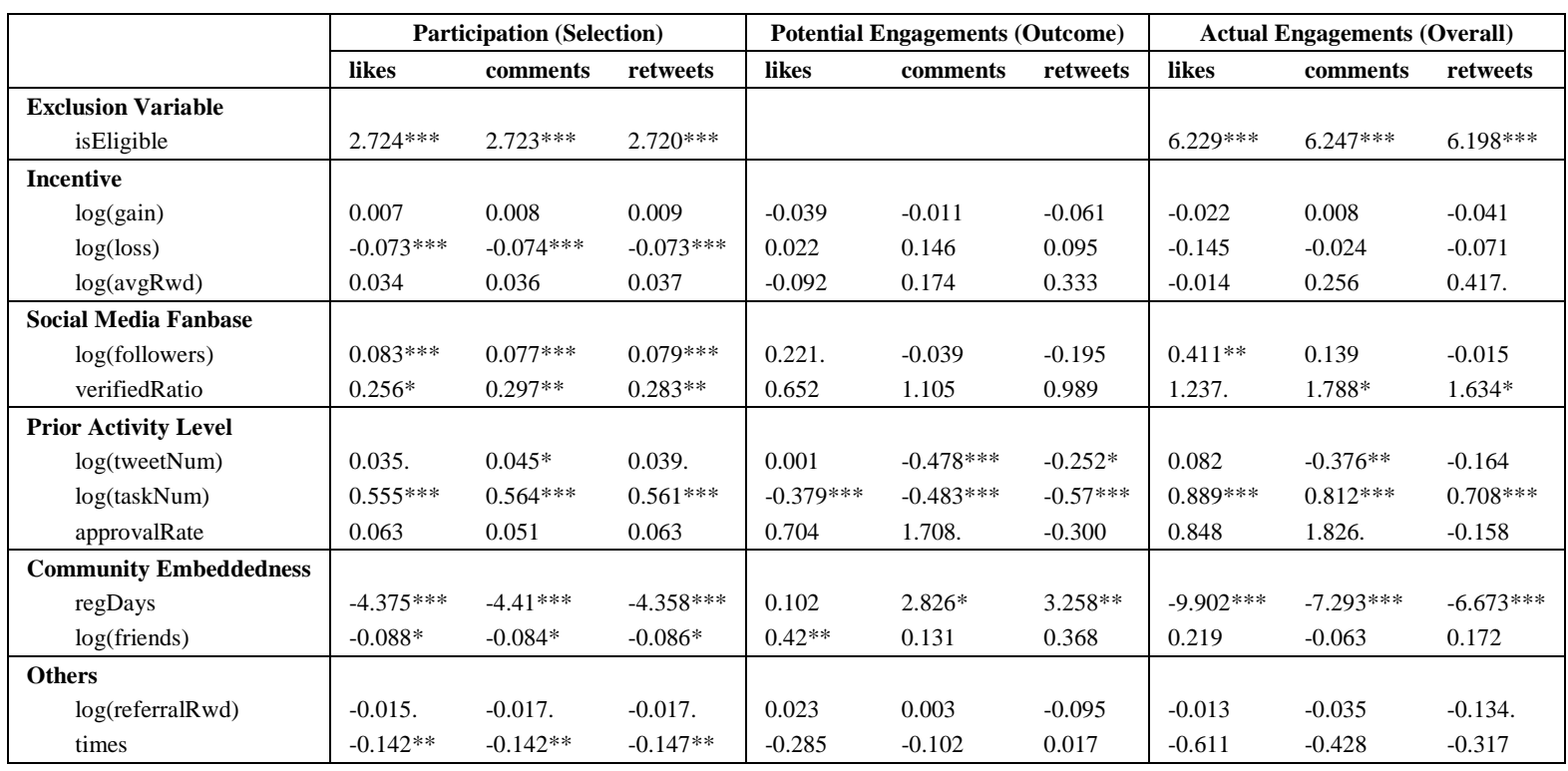

For the majority of predictors, the direction of the total effects is consistent with that in the participation equation. Hence, participation is oftentimes the primary driver of actual engagements. Variables for which this holds include the number of followers, the verified ratio, the task number, and the number of days since registration. As a concrete example, though having participated in many campaigns is associated with low potential in generating engagements, such endorsers tend to generate an above-average number of actual engagements, due to their high tendency to participate.

However, participation doesn't always dominate potential. For example, the direction of the total effect of the number of tweets is more consistent with its direction in the outcome equation, rather than the selection equation. The effect size of tweet number in the selection equation is small compared to that in the outcome equation. This finding is not surprising once one realizes that the mean value of $c_{i t}$ in equation (8) is 2.3 in our dataset. In some cases, the opposite effects in the participation and outcome equations may 
cancel out in the total effects, such as the total effect of the number of weituitui friends. These findings suggest that neglecting either participation or potential effectiveness in marketing campaigns can result in wrong decisions to target particular kinds of endorsers.

Advertisers unable to increase the participation or effectiveness of given endorsers may want to target endorsers who are likely to generate actual engagements, such as those who have participated in many tasks previously, have large fan bases, or a high verified ratio.

\section{Conclusions}

Paid endorsement, as an affordable approach to social advertising, has gained popularity among small firms in recent years. However, little is known on how to effectively target and incent paid endorsers. This paper provides new insights on how incentives and endorser characteristics affect participation and effectiveness. We conduct a field experiment on one of the largest paid endorsement platforms in China. For identification purpose, we exogenously manipulated the incentive and eligibility for participation. In order to analyze the collected panel count data on customer engagements while accounting for self-selection and repeated observations, we propose an approach that can address both challenges simultaneously.

Four findings have important implications for paid endorsement campaigns. First, endorsers are sensitive to losses but not gains as compared to their average reward in the past. This means that providing financial incentives in excess to an endorser's average reward per task in the past is very likely a waste of money. Advertisers should give attention to other aspects of the campaigns, such as non-monetary motives and the content of the ad message.

Second, the propensity to participate and effectiveness in generating engagements are often at odds with each other. This is so for both observed and unobserved characteristics. Consequently, it is difficult to find endorsers who are both responsive and effective. Advertisers should explore ways identify eligibility requirements that attract endorsers who are effective or find ways to boost the effort and effectiveness of endorsers who are responsive but otherwise ineffective. This may involve offering tasks with different requirements, eligibility restrictions or ad messages to different types of endorsers.

Third, it is misleading to assess the quality of endorsers solely based on the observed (actual) engagements. Endorsers observed to generate high engagements are not necessarily the most effective, but may simply be the most likely to participate. Conversely, the most effective endorsers tend not to participate in campaigns very often. This type of "latent gold" endorsers may easily be overlooked by marketers who do not distinguish between participation and effectiveness, or between actual and potential effectiveness.

Finally, which endorsers to target depends on the objectives of marketers. Some endorser characteristics are associated with generating higher-effort engagements such as comments and retweets, whereas other are associated with lower-effort engagements such as likes. 
Our work opens up several interesting directions for future research. First, it would be useful to study the effectiveness of endorsers in generating sales. Unfortunately, sales are hard to track at the individual level. Currently, the standard way to track clicks and sales is to use different short URLs in different tweets (even if they are for the same product), such that the source of clicks and sales can be tracked back to the short URLs. This means that clicks and sales can only be monitored at the level of task rather than endorser. More fine-grained tracking techniques are needed to study the effectiveness of individual endorsers in generating clicks and sales.

Secondly, our findings suggest that it would be useful to investigate the cost-effectiveness of different types of endorsers. This may require varying incentives or pay rates over a broad range to get robust insights. It may also be interesting to study how the effectiveness of various incentive and targeting approaches vary across product categories that vary in the status enhancement they provide to endorsers, such as mass vs. niche products or utilitarian vs. hedonic products.

Finally, the composition of the original message posted by the advertiser may also be worth investigating, as effective copy needs to appeal both to endorsers and to their followers. Here again, the distinction between participation and effectiveness may be essential to generating new fine-grained insights.

\section{Reference}

Abramowitz, M., and Stegun, I.A. 1972. Handbook of Mathematical Functions. Dover New York.

Alexandrov, A., Lilly, B., and Babakus, E. 2013. "The Effects of Social- and Self-Motives on the Intentions to Share Positive and Negative Word of Mouth," Journal of the Academy of Marketing Science (41:5), pp. 531-546.

Aral, S., and Walker, D. 2012. "Identifying Influential and Susceptible Members of Social Networks," Science (337:6092), pp. 337-341.

Aral, S., and Walker, D. 2014. "Tie Strength, Embeddedness, and Social Influence: A Large-Scale Networked Experiment," Management Science (60:6), pp. 1352-1370.

Barasch, A., and Berger, J. 2014. "Broadcasting and Narrowcasting: How Audience Size Affects What People Share," Journal of Marketing Research (51:3), pp. 286-299.

Berndt, E.K., Hall, B.H., and Hall, R.E. 1974. "Estimation and Inference in Nonlinear Structural Models," Annals of Economic and Social Measurement (3:4), pp. 653-665.

Bock, G.-W., Zmud, R.W., Kim, Y.-G., and Lee, J.-N. 2005. "Behavioral Intention Formation in Knowledge Sharing: Examining the Roles of Extrinsic Motivators, Social-Psychological Forces, and Organizational Climate," Management Information Systems Quarterly (29:1), pp. 87-111.

Brewer, M.B. 1976. "Randomized Invitations: One Solution to the Problem of Voluntary Treatment Selection in Program Evaluation Research," Social Science Research (5:3), pp. 315-323.

Burke, M. 2011. "Reading, Writing, Relationships: The Impact of Social Network Sites on Relationships and Well-Being," in: Human-Computer Interaction Institute. Carnegie Mellon University.

Butler, J.S., and Moffitt, R. 1982. "A Computationally Efficient Quadrature Procedure for the One-Factor Multinomial Probit Model," Econometrica (50:3), pp. 761-764.

Byrd, R.H., Lu, P., Nocedal, J., and Zhu, C. 1995. "A Limited Memory Algorithm for Bound Constrained Optimization," SIAM Journal on Scientific Computing (16:5), pp. 1190-1208. 
Cantor, D., O'Hare, B.C., and O'Connor, K.S. 2008. "The Use of Monetary Incentives to Reduce Nonresponse in Random Digit Dial Telephone Surveys," in Advances in Telephone Survey Methodology. pp. 471-498.

Chu, S.-C., and Kim, Y. 2011. "Determinants of Consumer Engagement in Electronic Word-of-Mouth (Ewom) in Social Networking Sites," International Journal of Advertising (30:1), pp. 47-75.

Cohn, A., Fehr, E., and Goette, L. 2015. "Fair Wages and Effort Provision: Combining Evidence from a Choice Experiment and a Field Experiment," Management Science (61:8), pp. 1777-1794.

Duflo, E., and Saez, E. 2003. "The Role of Information and Social Interactions in Retirement Plan Decisions: Evidence from a Randomized Experiment," Quarterly Journal of Economics (118:3), pp. 815-842.

Goel, S., Anderson, A., Hofman, J., and Watts, D.J. 2015. "The Structural Virality of Online Diffusion," Management Science (forthcoming).

Granovetter, M.S. 1973. "The Strength of Weak Ties," American Journal of Sociology (78:6), pp. 13601380.

Greene, W. 2009. "Models for Count Data with Endogenous Participation," Empirical Economics (36:1), pp. 133-173.

Greenleaf, E.A. 1995. "The Impact of Reference Price Effects on the Profitability of Price Promotions," Marketing Science (14:1), pp. 82-104.

Hall, D.B. 2000. "Zero-Inflated Poisson and Binomial Regression with Random Effects: A Case Study," Biometrics (56:4), pp. 1030-1039.

Hardie, B.G., Johnson, E.J., and Fader, P.S. 1993. "Modeling Loss Aversion and Reference Dependence Effects on Brand Choice," Marketing Science (12:4), pp. 378-394.

Heckman, J.J. 1979. "Sample Selection Bias as a Specification Error," Econometrica (47:1), pp. 153-161.

Ipeirotis, P.G., Provost, F., and Wang, J. 2010. "Quality Management on Amazon Mechanical Turk," in: Proceedings of the ACM SIGKDD Workshop on Human Computation. Washington DC: ACM, pp. 64-67.

Jones, S.C. 1973. "Self-and Interpersonal Evaluations: Esteem Theories Versus Consistency Theories," Psychological Bulletin (79:3), p. 185.

Kahneman, D., and Tversky, A. 1979. "Prospect Theory: An Analysis of Decision under Risk," Econometrica (47:2), pp. 263-291.

Kalyanaram, G., and Winer, R.S. 1995. "Empirical Generalizations from Reference Price Research," Marketing Science (14:3_supplement), pp. G161-G169.

Katona, Z., Zubcsek, P.P., and Sarvary, M. 2011. "Network Effects and Personal Influences: The Diffusion of an Online Social Network," Journal of Marketing Research (48:3), pp. 425-443.

King, R.A., Racherla, P., and Bush, V.D. 2014. "What We Know and Don't Know About Online Word-ofMouth: A Review and Synthesis of the Literature," Journal of Interactive Marketing (28:3), pp. 167183.

Kruskal, W. 1968. "When Are Gauss-Markov and Least Squares Estimators Identical? A Coordinate-Free Approach," The Annals of Mathematical Statistics (39:1), pp. 70-75.

Lattin, J.M., and Bucklin, R.E. 1989. "Reference Effects of Price and Promotion on Brand Choice Behavior," Journal of Marketing Research (26:3), pp. 299-310.

Long, X., and Nasiry, J. 2015. "Prospect Theory Explains Newsvendor Behavior: The Role of Reference Points," Management Science (forthcoming).

Lovett, M.J., Peres, R., and Shachar, R. 2013. "On Brands and Word of Mouth," Journal of Marketing Research (50:4), pp. 427-444.

Minkler, M. 2012. Community Organizing and Community Building for Health and Welfare. Rutgers University Press.

Moldoveanu, M.C., and Baum, J.A.C. 2011. "II Think You Think I Think You're Lying": The Interactive Epistemology of Trust in Social Networks," Management Science (57:2), pp. 393-412.

Paolacci, G., Chandler, J., and Ipeirotis, P.G. 2010. "Running Experiments on Amazon Mechanical Turk," Judgment and Decision making (5:5), pp. 411-419. 
Porter, S.R., and Whitcomb, M.E. 2003. "The Impact of Contact Type on Web Survey Response Rates," Public Opinion Quarterly (67:4), pp. 579-588.

Powers, D.E., and Swinton, S.S. 1984. "Effects of Self-Study for Coachable Test Item Types," Journal of Educational Psychology (76:2), pp. 266-278.

Puhani, P. 2000. "The Heckman Correction for Sample Selection and Its Critique," Journal of Economic Surveys (14:1), pp. 53-68.

Roberts, S.G.B., Dunbar, R.I.M., Pollet, T.V., and Kuppens, T. 2009. "Exploring Variation in Active Network Size: Constraints and Ego Characteristics," Social Networks (31:2), pp. 138-146.

Schau, H.J., and Gilly, M.C. 2003. "We Are What We Post? Self-Presentation in Personal Web Space," Journal of Consumer Research (30:3), pp. 385-404.

Shi, Z., Rui, H., and Whinston, A.B. 2014. "Content Sharing in a Social Broadcasting Environment: Evidence from Twitter," Management Information Systems Quarterly (38:1), pp. 123-142.

Singer, E., and Ye, C. 2013. "The Use and Effects of Incentives in Surveys," The Annals of the American Academy of Political and Social Science (645:1), pp. 112-141.

Toubia, O., and Stephen, A.T. 2013. "Intrinsic Vs. Image-Related Utility in Social Media: Why Do People Contribute Content to Twitter?," Marketing Science (32:3), pp. 368-392.

Trusov, M., Bodapati, A.V., and Bucklin, R.E. 2010. "Determining Influential Users in Internet Social Networks," Journal of Marketing Research (47:4), pp. 643-658.

Weenig, M.W., and Midden, C.J. 1991. "Communication Network Influences on Information Diffusion and Persuasion," Journal of Personality and Social Psychology (61:5), p. 734.

Weimann, G. 1983. "The Strength of Weak Conversational Ties in the Flow of Information and Influence," Social Networks (5:3), pp. 245-267.

Winkelmann, R. 1998. "Count Data Models with Selectivity," Econometric Reviews (17:4), pp. 339-359.

Yoganarasimhan, H. 2012. "Impact of Social Network Structure on Content Propagation: A Study Using Youtube Data," Quantitative Marketing and Economics (10:1), pp. 111-150.

Zhu, C., Byrd, R.H., Lu, P., and Nocedal, J. 1997. "Algorithm 778: L-Bfgs-B: Fortran Subroutines for Large-Scale Bound-Constrained Optimization," ACM Transactions on Mathematical Software (23:4), pp. 550-560. 


\section{Appendix A: Likelihood and Parameter Estimation}

The likelihood of all observations on endorser $i$ is given by

$$
\begin{gathered}
L_{i}=P\left(y_{i 1}^{*}, \ldots, y_{i T}^{*} ; z_{i 1}, \ldots, z_{i T} \mid \boldsymbol{x}_{i 1}, \ldots, \boldsymbol{x}_{i T}, \boldsymbol{w}_{i 1}, \ldots, \boldsymbol{w}_{i T}\right) \\
=\int_{-\infty}^{\infty} \phi\left(\varepsilon_{i}\right) d \varepsilon_{i} \int_{-\infty}^{\infty} f\left(u_{i} \mid \varepsilon_{i}\right) d u_{i} \int_{-\infty}^{\infty} P\left(y_{i 1}^{*}, \ldots, y_{i T}^{*} ; z_{i 1}, \ldots, z_{i T} \mid \boldsymbol{x}_{i 1}, \ldots, \boldsymbol{x}_{i T}, \boldsymbol{w}_{i 1}, \ldots, \boldsymbol{w}_{i T}, \varepsilon_{i}, u_{i}, \epsilon_{i 1}, \ldots, \epsilon_{i T}\right) \phi\left(\epsilon_{i 1}\right) \ldots \phi\left(\epsilon_{i T}\right) d \epsilon_{i 1} \ldots d \epsilon_{i T}
\end{gathered}
$$

Based on the i.i.d. assumption on the error terms $\epsilon_{i 1}, \ldots, \epsilon_{i T}$, the above likelihood can be simplified as

$$
\begin{gathered}
L_{i}=\int_{-\infty}^{\infty} \phi\left(\varepsilon_{i}\right) d \varepsilon_{i} \int_{-\infty}^{\infty} f\left(u_{i} \mid \varepsilon_{i}\right) d u_{i} \prod_{t=1}^{T_{i}} \int_{-\infty}^{\infty} P\left(y_{i t}^{*} \mid \boldsymbol{x}_{i t}, \varepsilon_{i}, \epsilon_{i t}\right)^{z_{i t}} P\left(z_{i t} \mid \boldsymbol{w}_{i t}, u_{i}, \epsilon_{i t}\right) \phi\left(\epsilon_{i t}\right) d \epsilon_{i t} \\
=\frac{1}{2 \pi \sqrt{1-\rho^{2}}} \int_{-\infty}^{\infty} e^{-\frac{\varepsilon_{i}^{2}}{2}} d \varepsilon_{i} \int_{-\infty}^{\infty} e^{-\frac{\left(u_{i}-\rho \varepsilon_{i}\right)^{2}}{2\left(1-\rho^{2}\right)}} d u_{i} \prod_{t=1}^{T_{i}} \int_{-\infty}^{\infty} \frac{1}{\sqrt{2 \pi}} P\left(y_{i t}^{*} \mid \boldsymbol{x}_{i t}, \varepsilon_{i}, \epsilon_{i t}\right)^{z_{i t}} \Phi\left(\left(2 z_{i t}-1\right) \frac{\boldsymbol{\alpha} \boldsymbol{w}_{i t}^{\prime}+\delta u_{i}+\tau \epsilon_{i t}}{\sqrt{1-\tau^{2}}}\right) e^{-\frac{\epsilon_{i t}^{2}}{2}} d \epsilon_{i t}
\end{gathered}
$$

After substituting in $r=\frac{\varepsilon_{i}}{\sqrt{2}}, s=\frac{u_{i}-\rho \varepsilon_{i}}{\sqrt{2\left(1-\rho^{2}\right)}}$, and $v=\frac{\epsilon_{i t}}{\sqrt{2}}$, the likelihood can be further simplified as

$$
L_{i}=\frac{1}{\pi} \int_{-\infty}^{\infty} e^{-r^{2}} d r \int_{-\infty}^{\infty} e^{-s^{2}} d s \prod_{t=1}^{T_{i}} \frac{1}{\sqrt{\pi}} \int_{-\infty}^{\infty} P\left(y_{i t}^{*} \mid x_{i t}, \sqrt{2} r, \sqrt{2} v\right)^{z_{i t}} \Phi\left(\left(2 z_{i t}-1\right) \frac{\alpha w_{i t}^{\prime}+\sqrt{2} \rho \delta r+\sqrt{2\left(1-\rho^{2}\right)} \delta s+\sqrt{2} \tau v}{\sqrt{1-\tau^{2}}}\right) e^{-v^{2}} d v
$$

In this form, the likelihood can be approximated numerically by Gauss-Hermite quadrature (Abramowitz and Stegun 1972; Greene 2009). Using the Gauss-Hermite quadrature procedure three times, the likelihood can be approximated as

$$
L_{i} \approx \frac{1}{\pi} \sum_{h=1}^{H} \omega_{h} \sum_{k=1}^{K} \varphi_{k} \prod_{t=1}^{T_{i}} \sum_{m=1}^{M} \frac{\mu_{m}}{\sqrt{\pi}} P\left(y_{i t}^{*} \mid \boldsymbol{x}_{i t}, \sqrt{2} r, \sqrt{2} v\right)^{z_{i t}} \Phi\left(\left(2 z_{i t}-1\right) \frac{\boldsymbol{\alpha} \boldsymbol{w}_{i t}^{\prime}+\sqrt{2} \rho \delta r_{h}+\sqrt{2\left(1-\rho^{2}\right)} \delta s_{k}+\sqrt{2} \tau v_{m}}{\sqrt{1-\tau^{2}}}\right)
$$

where $\left\{w_{h}, r_{h}\right\},\left\{\varphi_{k}, s_{k}\right\},\left\{\mu_{m}, v_{m}\right\}$ represent the weights and nodes for the three quadratures, with $H, K$, and $M$ points being used respectively. In our analysis, we find that $H=K=M=10$ are sufficient to yield reasonably good approximations.

The overall log likelihood on the entire dataset can be written as

$$
L L=\ln \prod_{i=1}^{N} L_{i}=\sum_{i=1}^{N} \ln L_{i}
$$

Since $\rho, \tau \in[-1,1]$, we use the L-BFGS-B method (Byrd et al. 1995; Zhu et al. 1997), which allows for box constraints, to maximize the log likelihood. We have found from simulations that the above likelihood maximization procedure can recover the true parameters very well.

Due to the complexity of the log likelihood, the asymptotic covariance matrix of the parameter estimates is computed using the BHHH estimator (Berndt et al. 1974; Greene 2009), which only requires the computation of the score function (i.e., gradient of log likelihood). 
Let $\boldsymbol{\theta}=[\boldsymbol{\alpha}, \delta, \rho, \tau]$ and $\boldsymbol{\eta}=[\boldsymbol{\beta}, \sigma, \gamma]$, the gradient of the log likelihood is approximated by

$$
\frac{\partial L L}{\partial \boldsymbol{\theta}} \approx \frac{1}{\pi} \sum_{i=1}^{N} \frac{1}{L_{i}} \sum_{h=1}^{H} \omega_{h} \sum_{k=1}^{K} \varphi_{k}\left(\prod_{t=1}^{T_{i}} \sum_{m=1}^{M} \frac{\mu_{m}}{\sqrt{\pi}} P\left(y_{i t} \mid \lambda_{i t}^{h m}\right)^{z_{i t}} \Phi\left(q_{i t}^{h k m}\right)\right) \sum_{t=1}^{T_{i}} \frac{\sum_{m=1}^{M} \frac{\mu_{m}}{\sqrt{\pi}} P\left(y_{i t} \mid \lambda_{i t}^{h m}\right)^{z_{i t}} \phi\left(q_{i t}^{h k m}\right) \frac{2 z_{i t}-1}{\sqrt{1-\tau^{2}}} \boldsymbol{w}_{i t}^{\text {ext }}}{\sum_{m=1}^{M} \frac{\mu_{m}}{\sqrt{\pi}} P\left(y_{i t} \mid \lambda_{i t}^{h m}\right)^{z_{i t}} \Phi\left(q_{i t}^{h k m}\right)}
$$

where $\boldsymbol{w}_{i t}^{\text {ext }}=\left[\boldsymbol{w}_{i t}, \sqrt{2} \rho r_{h}+\sqrt{2\left(1-\rho^{2}\right)} S_{k}, \sqrt{2} \delta r_{h}-\sqrt{\frac{2}{\left(1-\rho^{2}\right)}} \rho \delta s_{k}, \frac{\sqrt{2} v_{m}+\left(\boldsymbol{\alpha} \boldsymbol{w}_{i t}^{\prime}+\sqrt{2} \rho \delta r_{h}+\sqrt{2\left(1-\rho^{2}\right)} \delta s_{k}\right) \tau}{1-\tau^{2}}\right]$

$$
\frac{\partial L L}{\partial \boldsymbol{\eta}} \approx \frac{1}{\pi} \sum_{i=1}^{N} \frac{1}{L_{i}} \sum_{h=1}^{H} \omega_{h} \sum_{k=1}^{K} \varphi_{k}\left(\prod_{t=1}^{T_{i}} \sum_{m=1}^{M} \frac{\mu_{m}}{\sqrt{\pi}} P\left(y_{i t} \mid \lambda_{i t}^{h m}\right)^{z_{i t}} \Phi\left(q_{i t}^{h k m}\right)\right) \sum_{t=1}^{T_{i}} \frac{\sum_{m=1}^{M} z_{i t} \frac{\mu_{m}}{\sqrt{\pi}} P\left(y_{i t} \mid \lambda_{i t}^{h m}\right)\left(y_{i t}-\lambda_{i t}^{h m}\right) \Phi\left(q_{i t}^{h k m}\right) \boldsymbol{x}_{i t}^{\text {ext }}}{\sum_{m=1}^{M} \frac{\mu_{m}}{\sqrt{\pi}} P\left(y_{i t} \mid \lambda_{i t}^{h m}\right)^{z_{i t}} \Phi\left(q_{i t}^{h k m}\right)}
$$

where $\boldsymbol{x}_{i t}^{\text {ext }}=\left[\boldsymbol{x}_{i t}, \sqrt{2} r_{h}, \sqrt{2} v_{m}\right]$.

\section{Appendix B: Mean Actual Outcome $E\left[y_{i t} \mid \boldsymbol{x}_{i t}, \boldsymbol{w}_{i t}\right]$}

The unconditional (not conditional on any unobserved variable value) mean of actual outcome can be derived as follows

$$
\begin{aligned}
& E\left[y_{i t} \mid \boldsymbol{x}_{i t}, \boldsymbol{w}_{i t}\right]=E_{u_{i}} E_{\varepsilon_{i} \mid u_{i}} E_{\epsilon_{i t}}\left[E\left[y_{i t} \mid \boldsymbol{x}_{i t}, \boldsymbol{w}_{i t}, u_{i}, \varepsilon_{i}, \epsilon_{i t}\right]\right] \\
& =E_{u_{i}} E_{\varepsilon_{i} \mid u_{i}} E_{\epsilon_{i t}}\left[P\left(z_{i t}=1 \mid \boldsymbol{w}_{i t}, u_{i}, \epsilon_{i t}\right) E\left[y_{i t}^{*} \mid \boldsymbol{x}_{i t}, \varepsilon_{i}, \epsilon_{i t}\right]\right] \\
& =\int_{-\infty}^{\infty} \phi\left(u_{i}\right) d u_{i} \int_{-\infty}^{\infty} f\left(\varepsilon_{i} \mid u_{i}\right) d \varepsilon_{i} \int_{-\infty}^{\infty} \Phi\left(\frac{\boldsymbol{\alpha} \boldsymbol{w}_{i t}^{\prime}+\delta u_{i}+\tau \epsilon_{i t}}{\sqrt{1-\tau^{2}}}\right) \exp \left(\boldsymbol{\beta} \boldsymbol{x}_{i t}^{\prime}+\sigma \varepsilon_{i}+\gamma \epsilon_{i t}\right) \phi\left(\epsilon_{i t}\right) d \epsilon_{i t} \\
& =\exp \left(\boldsymbol{\beta} \boldsymbol{x}_{i t}^{\prime}\right) \int_{-\infty}^{\infty}\left[\int_{-\infty}^{\infty} \Phi\left(\frac{\boldsymbol{\alpha} \boldsymbol{w}_{i t}^{\prime}+\delta u_{i}+\tau \epsilon_{i t}}{\sqrt{1-\tau^{2}}}\right) \exp \left(\gamma \epsilon_{i t}\right) \phi\left(\epsilon_{i t}\right) d \epsilon_{i t}\right] \phi\left(u_{i}\right) d u_{i} \int_{-\infty}^{\infty} \exp \left(\sigma \varepsilon_{i}\right) f\left(\varepsilon_{i} \mid u_{i}\right) d \varepsilon_{i} \\
& =\exp \left(\boldsymbol{\beta} \boldsymbol{x}_{i t}^{\prime}\right) \int_{-\infty}^{\infty}\left[\int_{-\infty}^{\infty} \Phi\left(\frac{\boldsymbol{\alpha} \boldsymbol{w}_{i t}^{\prime}+\delta u_{i}+\tau \epsilon_{i t}}{\sqrt{1-\tau^{2}}}\right) \exp \left(\gamma \epsilon_{i t}\right) \phi\left(\epsilon_{i t}\right) d \epsilon_{i t}\right] \phi\left(u_{i}\right) d u_{i} \int_{-\infty}^{\infty} \frac{1}{\sqrt{2 \pi\left(1-\rho^{2}\right)} e^{\rho \sigma u_{i}+\frac{\left(1-\rho^{2}\right) \sigma^{2}}{2}} e^{-\frac{\left(\varepsilon_{i}-\rho u_{i}-\left(1-\rho^{2}\right) \sigma\right)^{2}}{2\left(1-\rho^{2}\right)}} d \varepsilon_{i}} \\
& =\exp \left(\boldsymbol{\beta} \boldsymbol{x}_{i t}^{\prime}\right) \int_{-\infty}^{\infty}\left[\int_{-\infty}^{\infty} \Phi\left(\frac{\boldsymbol{\alpha} \boldsymbol{w}_{i t}^{\prime}+\delta u_{i}+\tau \epsilon_{i t}}{\sqrt{1-\tau^{2}}}\right) \exp \left(\gamma \epsilon_{i t}\right) \phi\left(\epsilon_{i t}\right) d \epsilon_{i t}\right] e^{\rho \sigma u_{i}+\frac{\left(1-\rho^{2}\right) \sigma^{2}}{2}} \phi\left(u_{i}\right) d u_{i} \\
& =\frac{1}{2 \pi} \exp \left(\boldsymbol{\beta} \boldsymbol{x}_{i t}^{\prime}+\frac{\sigma^{2}+\gamma^{2}}{2}\right) \int_{-\infty}^{\infty}\left[\int_{-\infty}^{\infty} \Phi\left(\frac{\boldsymbol{\alpha} \boldsymbol{w}_{i t}^{\prime}+\delta u_{i}+\tau \epsilon_{i t}}{\sqrt{1-\tau^{2}}}\right) e^{-\frac{\left(\epsilon_{i t}-\gamma\right)^{2}}{2}} d \epsilon_{i t}\right] e^{-\frac{\left(u_{i}-\rho \sigma\right)^{2}}{2}} d u_{i}
\end{aligned}
$$

Let $v=\frac{\epsilon_{i t}-\gamma}{\sqrt{2}}$ and $r=\frac{u_{i}-\rho \sigma}{\sqrt{2}}$, the above equation can be simplified as

$$
E\left[y_{i t} \mid \boldsymbol{x}_{i t}, \boldsymbol{w}_{i t}\right]=\frac{1}{\pi} \exp \left(\boldsymbol{\beta} \boldsymbol{x}_{i t}^{\prime}+\frac{\sigma^{2}+\gamma^{2}}{2}\right) \int_{-\infty}^{\infty}\left[\int_{-\infty}^{\infty} \Phi\left(\frac{\boldsymbol{\alpha} \boldsymbol{w}_{i t}^{\prime}+\sqrt{2} \delta r+\rho \sigma \delta+\tau \gamma+\sqrt{2} \tau v}{\sqrt{1-\tau^{2}}}\right) e^{-v^{2}} d v\right] e^{-r^{2}} d r
$$

Similar to the likelihood function, $E\left[y_{i t} \mid \boldsymbol{x}_{i t}, \boldsymbol{w}_{i t}\right]$ can be approximated by two embedded GaussHermite quadratures. 


$$
E\left[y_{i t} \mid \boldsymbol{x}_{i t}, \boldsymbol{w}_{i t}\right] \approx \frac{1}{\pi} \exp \left(\boldsymbol{\beta} \boldsymbol{x}_{i t}^{\prime}+\frac{\sigma^{2}+\gamma^{2}}{2}\right) \sum_{h=1}^{H} \omega_{h} \sum_{k=1}^{K} \varphi_{k} \Phi\left(\frac{\boldsymbol{\alpha} \boldsymbol{w}_{i t}^{\prime}+\sqrt{2} \delta r_{h}+\rho \sigma \delta+\tau \gamma+\sqrt{2} \tau v_{k}}{\sqrt{1-\tau^{2}}}\right)
$$

where $\left\{w_{h}, r_{h}\right\}$, and $\left\{\varphi_{k}, s_{k}\right\}$ represent the weights and nodes for the two quadratures, with $H$ and $K$ points being used, respectively.

\section{Appendix C: Relative Partial Effects of $E\left[y_{i t} \mid \boldsymbol{x}_{i t}, \boldsymbol{w}_{i t}\right]$}

Letting $q_{i t}^{h k}=\frac{\boldsymbol{\alpha} \boldsymbol{w}_{i t}^{\prime}+\sqrt{2} \delta r_{h}+\rho \sigma \delta+\tau \gamma+\sqrt{2} \tau v_{k}}{\sqrt{1-\tau^{2}}}$, the relative partial effects of $\boldsymbol{x}_{i t}$ and $\boldsymbol{w}_{i t}$ on the mean actual outcome are given by

$$
\begin{gathered}
\boldsymbol{g}_{\boldsymbol{w}_{i t}}=\frac{\partial \log E\left[y_{i t} \mid \boldsymbol{x}_{i t}, \boldsymbol{w}_{i t}\right]}{\partial \boldsymbol{w}_{i t}}=c_{i t} \boldsymbol{\alpha} \approx \frac{\sum_{h=1}^{H} \omega_{h} \sum_{k=1}^{K} \varphi_{k} \phi\left(q_{i t}^{h k}\right)}{\sqrt{1-\tau^{2}} \sum_{h=1}^{H} \omega_{h} \sum_{k=1}^{K} \varphi_{k} \Phi\left(q_{i t}^{h k}\right)} \boldsymbol{\alpha} \\
\boldsymbol{g}_{x_{i t}}=\frac{\partial \log E\left[y_{i t} \mid \boldsymbol{x}_{i t}, \boldsymbol{w}_{i t}\right]}{\partial \boldsymbol{x}_{i t}} \approx \boldsymbol{\beta} \\
\text { where } c_{i t}=\frac{\int_{-\infty}^{\infty}\left[\int_{-\infty}^{\infty} \phi\left(\frac{\boldsymbol{\alpha} \boldsymbol{w}_{i t}^{\prime}+\sqrt{2} \delta r+\rho \sigma \delta+\tau \gamma+\sqrt{2} \tau v}{\sqrt{1-\tau^{2}}}\right) e^{-v^{2}} d v\right] e^{-r^{2}} d r}{\sqrt{1-\tau^{2}} \int_{-\infty}^{\infty}\left[\int_{-\infty}^{\infty} \Phi\left(\frac{\alpha \boldsymbol{w}_{i t}^{\prime}+\sqrt{2} \delta r+\rho \sigma \delta+\tau \gamma+\sqrt{2} \tau v}{\sqrt{1-\tau^{2}}}\right) e^{-v^{2}} d v\right] e^{-r^{2}} d r}>0 .
\end{gathered}
$$

If a variable appears in both $\boldsymbol{x}_{i t}$ and $\boldsymbol{w}_{i t}$, the derivative for that variable would be the sum of the corresponding elements in $\boldsymbol{g}_{\boldsymbol{x}_{i t}}$ and $\boldsymbol{g}_{\boldsymbol{w}_{i t}}$.

The covariance matrix of the relative partial effects can be approximated by the delta method. Letting $\boldsymbol{\theta}=[\boldsymbol{\alpha}, \boldsymbol{\beta}, \delta, \sigma, \gamma, \rho, \tau], A=\sum_{h=1}^{H} \omega_{h} \sum_{k=1}^{K} \varphi_{k} \phi\left(q_{i t}^{h k}\right)$, and $B=\sum_{h=1}^{H} \omega_{h} \sum_{k=1}^{K} \varphi_{k} \Phi\left(q_{i t}^{h k}\right)$, the first order derivative of the relative partial effects w.r.t. the model parameters can be computed as

$$
\begin{gathered}
\boldsymbol{J}_{\boldsymbol{w}_{i t}}=\frac{\partial \boldsymbol{g}_{\boldsymbol{w}_{i t}}^{\prime}}{\partial \boldsymbol{\theta}}=\frac{A}{\sqrt{1-\tau^{2}} B} \frac{\partial \boldsymbol{\alpha}^{\prime}}{\partial \boldsymbol{\theta}}+\boldsymbol{\alpha}^{\prime} \frac{\partial \frac{A}{\sqrt{1-\tau^{2}} B}}{\partial \boldsymbol{\theta}} \\
=\frac{A}{\sqrt{1-\tau^{2}} B}\left[I_{|\boldsymbol{\alpha}| *|\boldsymbol{\alpha}|}, 0_{|\boldsymbol{\alpha}| *(|\boldsymbol{\beta}|+4)}, \frac{2 \tau}{1-\tau^{2}} \boldsymbol{\alpha}^{\prime}\right]+\boldsymbol{\alpha}^{\prime} \frac{\sum_{h=1}^{H} \omega_{h} \sum_{k=1}^{K}-q_{i t}^{h k} \varphi_{k} \phi\left(q_{i t}^{h k}\right) \frac{\partial q_{i t}^{h k}}{\partial \boldsymbol{\theta}}-\frac{A}{B} \sum_{h=1}^{H} \omega_{h} \sum_{k=1}^{K} \varphi_{k} \phi\left(q_{i t}^{h k}\right) \frac{\partial q_{i t}^{h k}}{\partial \boldsymbol{\theta}}}{\sqrt{1-\tau^{2}} B} \\
\text { where } \frac{\partial q_{i t}^{h k}}{\partial \boldsymbol{\theta}}=\frac{1}{\sqrt{1-\tau^{2}}}\left[\boldsymbol{w}_{i t}, 0_{|\boldsymbol{\beta}|}, \sqrt{2} r_{h}+\rho \sigma, \rho \delta, \tau, \sigma \delta, \frac{\left(\gamma+\sqrt{2} v_{k}\right)\left(1+\tau^{2}\right)+2 \tau\left(\boldsymbol{\alpha} \boldsymbol{w}_{i t}^{\prime}+\sqrt{2} \delta r_{h}+\rho \sigma \delta\right)}{1-\tau^{2}}\right] \\
\qquad \boldsymbol{J}_{i t}=\frac{\partial \boldsymbol{g}_{\boldsymbol{x}_{i t}}^{\prime}}{\partial \boldsymbol{\theta}}=\frac{\partial \boldsymbol{\beta}^{\prime}}{\partial \boldsymbol{\theta}}=\left[0_{|\boldsymbol{\beta}| *|\boldsymbol{\alpha}|}, I_{|\boldsymbol{\beta}| *|\boldsymbol{\beta}|}, 0_{|\boldsymbol{\beta}| * 5}\right]
\end{gathered}
$$

Similarly, if a variable appears in both $\boldsymbol{x}_{i t}$ and $\boldsymbol{w}_{i t}$, the derivative for that variable would be the sum of the corresponding rows in $\boldsymbol{J}_{\boldsymbol{x}_{i t}}$ and $\boldsymbol{J}_{\boldsymbol{w}_{i t}}$.

Letting $\boldsymbol{g}$ represent the relative partial effects of all variables, and $\boldsymbol{J}$ be the first order derivative of $\boldsymbol{g}$ w.r.t. the model parameters, then according to the delta method 


$$
\operatorname{Var}(\boldsymbol{g}) \approx \boldsymbol{J} \boldsymbol{V} \boldsymbol{J}^{\prime}
$$

where $\boldsymbol{V}$ is the covariance matrix of the parameter estimates.

\section{Appendix D: Prediction of An Endorser's Participation and Effectiveness in a Task}

1) Probability to participate

$$
\begin{aligned}
& P\left(z_{i t}=1 \mid \boldsymbol{w}_{i t}\right)=\int_{-\infty}^{\infty} P\left(z_{i t}=1 \mid \boldsymbol{w}_{i t}, u_{i}\right) \phi\left(u_{i}\right) d u_{i}=\int_{-\infty}^{\infty} \Phi\left(\boldsymbol{\alpha} \boldsymbol{w}_{i t}^{\prime}+\delta u_{i}\right) \phi\left(u_{i}\right) d u_{i} \\
& =\frac{1}{\sqrt{2 \pi}} \int_{-\infty}^{\infty} \Phi\left(\boldsymbol{\alpha} \boldsymbol{w}_{i t}^{\prime}+\delta u_{i}\right) e^{-\frac{u_{i}^{2}}{2}} d u_{i}=\frac{1}{\sqrt{\pi}} \int_{-\infty}^{\infty} \Phi\left(\boldsymbol{\alpha} \boldsymbol{w}_{i t}^{\prime}+\sqrt{2} \delta v\right) e^{-v^{2}} d v=\frac{1}{\sqrt{\pi}} \sum_{k=1}^{K} \omega_{k} \Phi\left(\boldsymbol{\alpha} \boldsymbol{w}_{i t}^{\prime}+\sqrt{2} \delta v\right)
\end{aligned}
$$

2) Expected number of engagements

$$
\begin{aligned}
& E\left[y_{i t} \mid \boldsymbol{x}_{i t}\right]=E_{\varepsilon_{i}} E_{\epsilon_{i t}}\left[E\left[y_{i t} \mid \boldsymbol{x}_{i t}, \varepsilon_{i}, \epsilon_{i t}\right]\right]=\int_{-\infty}^{\infty} \phi\left(\varepsilon_{i}\right) d \varepsilon_{i} \int_{-\infty}^{\infty} \exp \left(\boldsymbol{\beta} \boldsymbol{x}_{i t}^{\prime}+\sigma \varepsilon_{i}+\gamma \epsilon_{i t}\right) \phi\left(\epsilon_{i t}\right) d \epsilon_{i t} \\
& =\frac{1}{2 \pi} \int_{-\infty}^{\infty} e^{-\frac{\varepsilon_{i}^{2}}{2}} d \varepsilon_{i} \int_{-\infty}^{\infty} \exp \left(\boldsymbol{\beta} \boldsymbol{x}_{i t}^{\prime}+\sigma \varepsilon_{i}+\gamma \epsilon_{i t}\right) e^{-\frac{\epsilon_{i t}^{2}}{2}} d \epsilon_{i t} \\
& =\frac{1}{\pi} \int_{-\infty}^{\infty} e^{-u^{2}} d u \int_{-\infty}^{\infty} \exp \left(\boldsymbol{\beta} \boldsymbol{x}_{i t}^{\prime}+\sqrt{2} \sigma u+\sqrt{2} \gamma v\right) e^{-v^{2}} d v \\
& =\frac{1}{\pi} \sum_{h=1}^{H} \varphi_{h} \sum_{k=1}^{K} \omega_{k} \exp \left(\boldsymbol{\beta} \boldsymbol{x}_{i t}^{\prime}+\sqrt{2} \sigma u_{h}+\sqrt{2} \gamma v_{k}\right)
\end{aligned}
$$




\section{Online Appendix}

\section{A. Results Using Alternative Incentives}

Table OA.1 Parameter Estimates for Different Types of Engagements (Pay Rate)

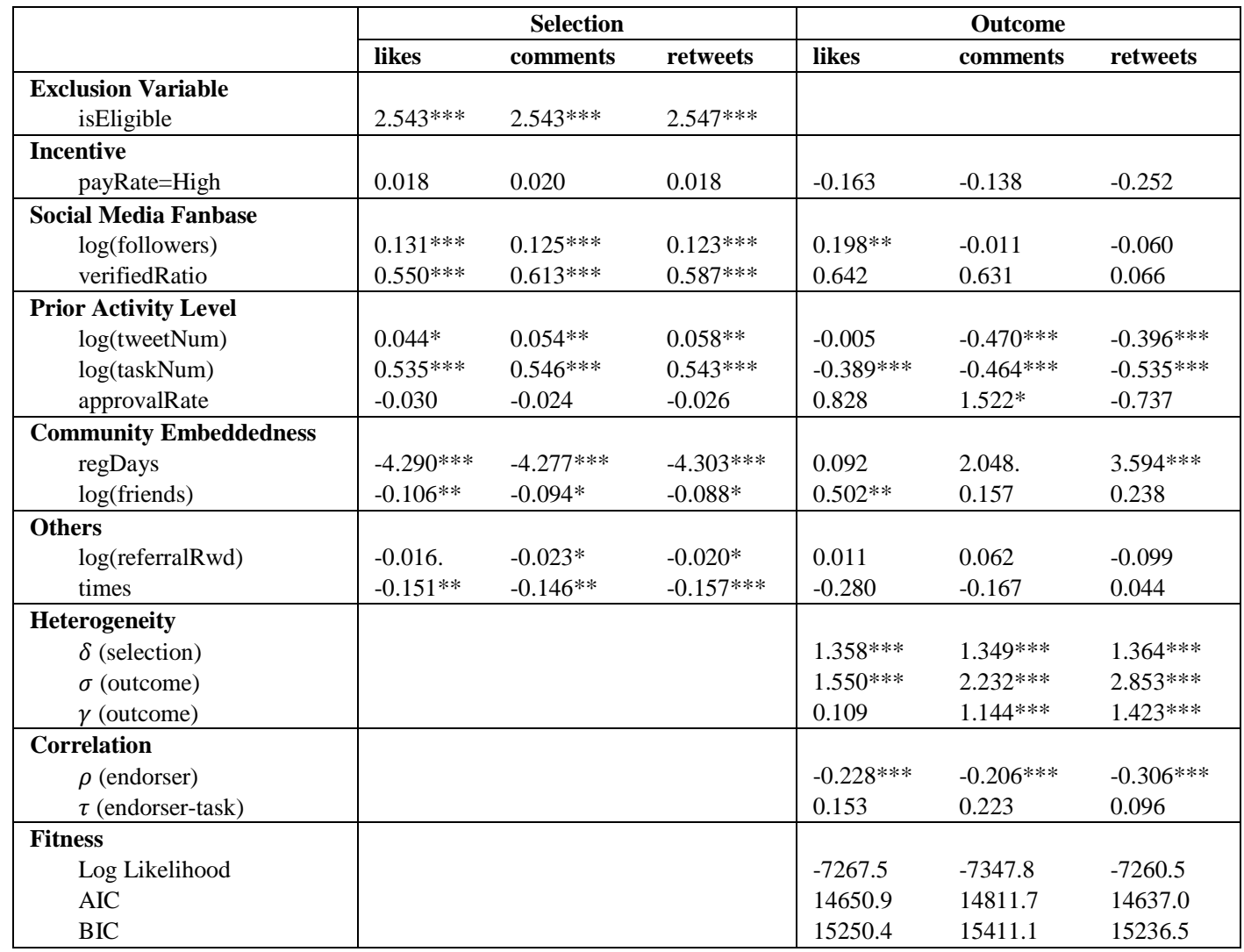


Table OA.2 Parameter Estimates for Different Types of Engagements (Linear Reward)

\begin{tabular}{|c|c|c|c|c|c|c|}
\hline & \multicolumn{3}{|c|}{ Selection } & \multicolumn{3}{|c|}{ Outcome } \\
\hline & likes & comments & retweets & likes & comments & retweets \\
\hline $\begin{array}{c}\text { Exclusion Variable } \\
\text { isEligible } \\
\end{array}$ & $2.535 * * *$ & $2.532 * * *$ & $2.528 * * *$ & & & \\
\hline $\begin{array}{r}\text { Incentive } \\
\text { actRwd }\end{array}$ & $0.011 *$ & $0.012 *$ & 0.011 . & 0.015 & 0.063 & $0.114 * * *$ \\
\hline $\begin{array}{c}\text { Social Media Fanbase } \\
\quad \log (\text { followers }) \\
\text { verifiedRatio } \\
\end{array}$ & $\begin{array}{l}0.123^{* * *} \\
0.507 * * * \\
\end{array}$ & $\begin{array}{l}0.120 * * * \\
0.552 * * * \\
\end{array}$ & $\begin{array}{l}0.121 * * * \\
0.559 * * *\end{array}$ & $\begin{array}{l}0.172^{*} \\
0.593 \\
\end{array}$ & $\begin{array}{l}-0.116 \\
0.628 \\
\end{array}$ & $\begin{array}{l}-0.150 \\
-0.051 \\
\end{array}$ \\
\hline $\begin{array}{c}\text { Prior Activity Level } \\
\log (\text { tweetNum }) \\
\log (\text { taskNum }) \\
\text { approvalRate }\end{array}$ & $\begin{array}{l}0.045^{*} \\
0.535^{* * *} \\
-0.023\end{array}$ & $\begin{array}{l}0.061 * * \\
0.547 * * * \\
-0.033\end{array}$ & $\begin{array}{l}0.048 * \\
0.549 * * * \\
-0.016\end{array}$ & $\begin{array}{l}-0.012 \\
-0.356^{* * * *} \\
0.764\end{array}$ & $\begin{array}{l}-0.408 * * * \\
-0.358 * * * \\
1.403\end{array}$ & $\begin{array}{l}-0.482 * * * \\
-0.674 * * * \\
-0.022\end{array}$ \\
\hline $\begin{array}{l}\text { Community Embeddedness } \\
\text { regDays } \\
\log (\text { friends }) \\
\end{array}$ & $\begin{array}{l}-4.284 * * * \\
-0.105 * * \\
\end{array}$ & $\begin{array}{l}-4.303 * * * \\
-0.107 * * \\
\end{array}$ & $\begin{array}{l}-4.251 * * * \\
-0.100 *\end{array}$ & $\begin{array}{l}-0.050 \\
0.452 * *\end{array}$ & $\begin{array}{l}2.244^{*} \\
0.099 \\
\end{array}$ & $\begin{array}{l}3.048^{*} \\
0.259 \\
\end{array}$ \\
\hline $\begin{array}{l}\text { Others } \\
\quad \log (\text { referralRwd) } \\
\text { times }\end{array}$ & $\begin{array}{l}-0.016 \\
-0.152 * *\end{array}$ & $\begin{array}{l}-0.022^{*} \\
-0.156^{* * *}\end{array}$ & $\begin{array}{l}-0.023^{*} \\
-0.152^{* * *}\end{array}$ & $\begin{array}{l}0.014 \\
-0.281\end{array}$ & $\begin{array}{l}0.035 \\
-0.169\end{array}$ & $\begin{array}{l}-0.043 \\
0.008\end{array}$ \\
\hline $\begin{array}{r}\text { Heterogeneity } \\
\delta \text { (selection) } \\
\sigma \text { (outcome) } \\
\gamma \text { (outcome) } \\
\end{array}$ & & & & $\begin{array}{l}1.358 * * * \\
1.564 * * * \\
0.005 \\
\end{array}$ & $\begin{array}{l}1.360 * * * \\
2.105 * * * \\
1.040 * * *\end{array}$ & $\begin{array}{l}1.359 * * * \\
2.795 * * * \\
1.412 * * \\
\end{array}$ \\
\hline $\begin{array}{l}\text { Correlation } \\
\rho \text { (endorser) } \\
\tau \text { (endorser-task) } \\
\end{array}$ & & & & $\begin{array}{l}-0.215^{* * *} \\
-0.104\end{array}$ & $\begin{array}{l}-0.297 * * * \\
0.319^{*}\end{array}$ & $\begin{array}{l}-0.252^{* * *} \\
0.269 *\end{array}$ \\
\hline $\begin{array}{l}\text { Fitness } \\
\text { Log Likelihood } \\
\text { AIC } \\
\text { BIC }\end{array}$ & & & & $\begin{array}{l}-7265.3 \\
14646.6 \\
15246.0\end{array}$ & $\begin{array}{l}-7343.7 \\
14803.3 \\
15402.8\end{array}$ & $\begin{array}{l}-7256.6 \\
14629.2 \\
15228.6\end{array}$ \\
\hline
\end{tabular}


Table OA.3 Parameter Estimates for Different Types of Engagements (Linear GainLoss)

\begin{tabular}{|c|c|c|c|c|c|c|}
\hline & \multicolumn{3}{|c|}{ Selection } & \multicolumn{3}{|c|}{ Outcome } \\
\hline & likes & comments & retweets & likes & comments & retweets \\
\hline $\begin{array}{c}\text { Exclusion Variable } \\
\text { isEligible } \\
\end{array}$ & $2.544 * * *$ & $2.539 * * *$ & $2.539 * * *$ & & & \\
\hline \begin{tabular}{l}
\multicolumn{1}{|l}{ Incentive } \\
$\quad$ gain \\
loss \\
avgRwd \\
\end{tabular} & $\begin{array}{l}-0.018 \\
-0.253^{* * *} \\
0.107^{*} \\
\end{array}$ & $\begin{array}{l}-0.018 \\
-0.245^{* * *} \\
0.102^{* *} \\
\end{array}$ & $\begin{array}{l}-0.018 \\
-0.237 * * * \\
0.102 * \\
\end{array}$ & $\begin{array}{l}-0.004 \\
0.108 \\
0.044 \\
\end{array}$ & $\begin{array}{l}0.090 \\
0.187 \\
0.048 \\
\end{array}$ & $\begin{array}{l}-0.064 \\
0.033 \\
0.250 * * \\
\end{array}$ \\
\hline $\begin{array}{l}\text { Social Media Fanbase } \\
\log \text { (followers) } \\
\text { verifiedRatio } \\
\end{array}$ & $\begin{array}{l}0.113 * * * \\
0.411 * * *\end{array}$ & $\begin{array}{l}0.107 * * * \\
0.457 * * *\end{array}$ & $\begin{array}{l}0.107 * * * \\
0.450 * * *\end{array}$ & $\begin{array}{l}0.157 * \\
0.775 \\
\end{array}$ & $\begin{array}{l}-0.189^{*} \\
0.643 \\
\end{array}$ & $\begin{array}{l}-0.155 \\
-0.191 \\
\end{array}$ \\
\hline $\begin{array}{c}\text { Prior Activity Level } \\
\log (\text { tweetNum }) \\
\log (\text { taskNum }) \\
\text { approvalRate } \\
\end{array}$ & $\begin{array}{l}0.042 * \\
0.539 * * * \\
-0.004 \\
\end{array}$ & $\begin{array}{l}0.051 * \\
0.554 * * * \\
-0.008 \\
\end{array}$ & $\begin{array}{l}0.050 * \\
0.538 * * * \\
0.013 \\
\end{array}$ & $\begin{array}{l}0.026 \\
-0.366^{* * * *} \\
0.601 \\
\end{array}$ & $\begin{array}{l}-0.257^{*} \\
-0.453^{* * *} \\
1.340 \\
\end{array}$ & $\begin{array}{l}-0.480 * * * \\
-0.386^{* *} \\
-0.892 \\
\end{array}$ \\
\hline $\begin{array}{l}\text { Community Embeddedness } \\
\text { regDays } \\
\log \text { (friends) } \\
\end{array}$ & $\begin{array}{l}-4.344 * * * \\
-0.098 * \\
\end{array}$ & $\begin{array}{l}-4.375 * * * \\
-0.099 * \\
\end{array}$ & $\begin{array}{l}-4.258 * * * \\
-0.087 *\end{array}$ & $\begin{array}{l}0.107 \\
0.399 *\end{array}$ & $\begin{array}{l}2.166 \\
-0.059 \\
\end{array}$ & $\begin{array}{l}3.134^{*} \\
0.205 \\
\end{array}$ \\
\hline $\begin{array}{l}\text { Others } \\
\begin{array}{l}\log (\text { referralRwd }) \\
\text { times }\end{array} \\
\end{array}$ & $\begin{array}{l}-0.015 \\
-0.154 * * *\end{array}$ & $\begin{array}{l}-0.021 * \\
-0.152 * * *\end{array}$ & $\begin{array}{l}-0.021 * \\
-0.152 * * \\
\end{array}$ & $\begin{array}{l}0.026 \\
-0.288 \\
\end{array}$ & $\begin{array}{l}0.027 \\
-0.218 \\
\end{array}$ & $\begin{array}{l}0.056 \\
0.015 \\
\end{array}$ \\
\hline $\begin{array}{r}\text { Heterogeneity } \\
\delta \text { (selection) } \\
\sigma \text { (outcome) } \\
\gamma \text { (outcome) }\end{array}$ & & & & $\begin{array}{l}1.353 * * * \\
1.578^{* * *} * \\
0\end{array}$ & $\begin{array}{l}1.359^{* * *} \\
2.240^{* * *} \\
1.175^{* * * *}\end{array}$ & $\begin{array}{l}1.339 * * * \\
2.846 * * * \\
1.447 * * *\end{array}$ \\
\hline $\begin{array}{l}\text { Correlation } \\
\quad \rho \text { (endorser) } \\
\tau \text { (endorser-task) } \\
\end{array}$ & & & & $\begin{array}{l}-0.240 * * * \\
0.005\end{array}$ & $\begin{array}{l}-0.300^{* * * *} \\
0.238\end{array}$ & $\begin{array}{l}-0.242 * * * \\
0.207 \\
\end{array}$ \\
\hline $\begin{array}{l}\text { Fitness } \\
\text { Log Likelihood } \\
\text { AIC } \\
\text { BIC } \\
\end{array}$ & & & & $\begin{array}{l}-7251.3 \\
14626.5 \\
15267.3\end{array}$ & $\begin{array}{l}-7329.4 \\
14782.8 \\
15423.6\end{array}$ & $\begin{array}{l}-7242.0 \\
14607.9 \\
15248.7 \\
\end{array}$ \\
\hline
\end{tabular}

The negative effects of follower number on comments and retweets in the outcome equation, though not well-supported in other models, are consistent with our reasoning that network size may have a negative effect on overall influence in higheffort behaviors (Katona et al. 2011). 
Table OA.4 Parameter Estimates for Different Types of Engagements (Log Reward)

\begin{tabular}{|c|c|c|c|c|c|c|}
\hline & \multicolumn{3}{|c|}{ Selection } & \multicolumn{3}{|c|}{ Outcome } \\
\hline & likes & comments & retweets & likes & comments & retweets \\
\hline $\begin{array}{c}\text { Exclusion Variable } \\
\text { isEligible } \\
\end{array}$ & 0.967 *** & $0.948 * * *$ & $0.952 * * *$ & & & \\
\hline $\begin{array}{c}\text { Financial Incentive } \\
\log (\mathrm{actRwd})\end{array}$ & $0.266^{* * *}$ & $0.267 * * *$ & $0.268 * * *$ & -0.085 & -0.010 & -0.110 \\
\hline $\begin{array}{c}\text { Social Media Fanbase } \\
\log (\text { followers }) \\
\text { verifiedRatio }\end{array}$ & $\begin{array}{l}0.017 \\
0.154\end{array}$ & $\begin{array}{l}0.007 \\
0.198\end{array}$ & $\begin{array}{l}0.008 \\
0.190\end{array}$ & $\begin{array}{l}0.202^{*} \\
0.646\end{array}$ & $\begin{array}{l}0.005 \\
0.806\end{array}$ & $\begin{array}{l}-0.081 \\
0.386\end{array}$ \\
\hline $\begin{array}{c}\text { Prior Activity Level } \\
\log \text { (tweetNum) } \\
\log \text { (taskNum) } \\
\text { approvalRate }\end{array}$ & $\begin{array}{l}0.047 * \\
0.543 * * * \\
-0.003\end{array}$ & $\begin{array}{l}0.056^{* *} \\
0.548^{* * *} \\
0.005\end{array}$ & $\begin{array}{l}0.053 * * \\
0.542 * * * \\
0.015\end{array}$ & $\begin{array}{l}0.006 \\
-0.354 * * \\
0.727\end{array}$ & $\begin{array}{l}-0.478 * * * \\
-0.414 * * * \\
1.638^{*}\end{array}$ & $\begin{array}{l}-0.491 * * * \\
-0.311 * * \\
-0.462\end{array}$ \\
\hline $\begin{array}{l}\text { Community Embeddedness } \\
\text { regDays } \\
\log (\text { friends }) \\
\end{array}$ & $\begin{array}{l}-4.295 * * * \\
-0.100 * *\end{array}$ & $\begin{array}{l}-4.255^{* * *} \\
-0.093^{*} \\
\end{array}$ & $\begin{array}{l}-4.211 * * * \\
-0.080 *\end{array}$ & $\begin{array}{l}-0.025 \\
0.389 *\end{array}$ & $\begin{array}{l}1.997 . \\
0.046\end{array}$ & $\begin{array}{l}3.130 * * \\
-0.062 \\
\end{array}$ \\
\hline $\begin{array}{l}\text { Others } \\
\text { promIncm } \\
\text { times } \\
\end{array}$ & $\begin{array}{l}-0.018^{*} \\
-0.142 * * \\
\end{array}$ & $\begin{array}{l}-0.022^{*} \\
-0.144 * *\end{array}$ & $\begin{array}{l}-0.023^{*} \\
-0.147 * *\end{array}$ & $\begin{array}{l}0.018 \\
-0.298 \\
\end{array}$ & $\begin{array}{l}0.030 \\
-0.176 \\
\end{array}$ & $\begin{array}{l}-0.033 \\
-0.127 \\
\end{array}$ \\
\hline $\begin{array}{r}\text { Heterogeneity } \\
\delta \text { (selection) } \\
\sigma \text { (outcome) } \\
\gamma \text { (outcome) } \\
\end{array}$ & & & & $\begin{array}{l}1.360 * * * \\
1.597 * * * \\
0.031\end{array}$ & $\begin{array}{l}1.348 * * * \\
2.257 * * * \\
1.124 * * *\end{array}$ & $\begin{array}{l}1.344 * * * \\
2.661 * * * \\
1.389 * * *\end{array}$ \\
\hline $\begin{array}{l}\text { Correlation } \\
\quad \rho \text { (endorser) } \\
\tau \text { (endorser-task) }\end{array}$ & & & & $\begin{array}{l}-0.224 * * * \\
-0.012\end{array}$ & $\begin{array}{l}-0.259 * * * \\
0.433 * *\end{array}$ & $\begin{array}{l}-0.242 * * * \\
0.380 * *\end{array}$ \\
\hline $\begin{array}{l}\text { Fitness } \\
\text { Log Likelihood } \\
\text { AIC } \\
\text { BIC }\end{array}$ & & & & $\begin{array}{l}-7243.6 \\
14603.2 \\
15202.7\end{array}$ & $\begin{array}{l}-7323.4 \\
14762.9 \\
15362.3\end{array}$ & $\begin{array}{l}-7239.2 \\
14594.4 \\
15193.8\end{array}$ \\
\hline
\end{tabular}

Due to the high correlation (0.98) between "isEligible" and "log(actRwd)", the parameter estimate on "isEligible" is very different from that in other models. 


\section{B. Robustness}

Table OA.5 Effect of Eligibility on Effort Level

\begin{tabular}{|l|l|l|}
\hline & \# Words in retweets & Use of emoji \\
\hline isEligible & -0.063 & -0.137 \\
\hline $\log$ (gain) & 0.010 & 0.039 \\
$\log (\operatorname{loss})$ & 0.007 & 0.071 \\
$\log$ (avgRwd) & $-0.130^{* * *}$ & -0.105 \\
$\log$ (followers) & $0.083^{* * *}$ & 0.136. \\
verifiedRatio & 0.139 & 0.493 \\
$\log$ (tweetNum) & -0.020 & 0.083 \\
$\log$ (taskNum) & $0.106^{* * *}$ & -0.114 \\
approvalRate & 0.120 & 0.455 \\
regDays & -0.018 & -1.207. \\
$\log$ (friends) & -0.030 & 0.168 \\
$\log$ (referralRwd) & 0.005 & -0.012 \\
times & 0.015 & -0.350 \\
$\delta$ & & $1.533 * * *$ \\
$\sigma$ & $0.814^{* * *}$ & \\
$\gamma$ & $0.448^{* * *}$ & \\
\hline Log Likelihood & -8406.1 & -591.8 \\
\hline
\end{tabular}

Since the number of words is a count variable and the use of emoji is binary, we use a Poisson Lognormal model with random effects on endorser level (similar to our outcome equation) and a Probit model with random effects on endorser level (similar to our selection equation) to estimate the effects of eligibility on these two effort metrics, respectively. 
Table OA.6 Robustness to Model Complexity

\begin{tabular}{|c|c|c|c|c|c|c|c|c|c|}
\hline & \multicolumn{3}{|c|}{ Full } & \multicolumn{3}{|c|}{ Without $\tau$} & \multicolumn{3}{|c|}{ Without $\tau \& \gamma$} \\
\hline & likes & comments & retweets & likes & comments & retweets & likes & comments & retweets \\
\hline \multicolumn{10}{|l|}{ Selection } \\
\hline isEligible & $2.724 * * *$ & $2.723 * * *$ & $2.720 * * *$ & $2.727 * * *$ & 2.730 *** & $2.732 * * *$ & $2.732 * * *$ & $2.719 * * *$ & $2.723 * * *$ \\
\hline $\log$ (gain) & 0.007 & 0.008 & 0.009 & 0.007 & 0.007 & 0.006 & 0.007 & 0.008 & 0.006 \\
\hline $\log (\operatorname{loss})$ & $-0.073 * * *$ & $-0.074 * * *$ & $-0.073 * * *$ & $-0.074 * * *$ & $-0.076^{* * * *}$ & $-0.075^{* * * *}$ & $-0.074 * * *$ & $-0.072 * * *$ & $-0.073 * * *$ \\
\hline $\log (\operatorname{avgRwd})$ & 0.034 & 0.036 & 0.037 & 0.039 & 0.038 & 0.036 & 0.040 & 0.024 & 0.029 \\
\hline $\log$ (followers) & $0.083 * * *$ & $0.077 * * *$ & $0.079 * * *$ & $0.082 * * *$ & $0.077 * * *$ & $0.077^{* * * *}$ & $0.082 * * *$ & $0.077 * * *$ & $0.077 * * *$ \\
\hline verifiedRatio & $0.256^{*}$ & $0.297 * *$ & $0.283 * *$ & $0.251^{*}$ & $0.302 * *$ & $0.299 * *$ & $0.260 *$ & $0.290 * *$ & $0.290 * *$ \\
\hline $\log ($ tweetNum) & 0.035 . & $0.045^{*}$ & 0.039 . & 0.035 . & $0.042 *$ & 0.036 . & 0.035 . & $0.041^{*}$ & 0.036 \\
\hline $\log (\operatorname{taskNum})$ & $0.555^{* * *}$ & $0.564 * * *$ & $0.561 * * *$ & $0.553 * * *$ & $0.560 * * *$ & $0.544 * * *$ & $0.554 * * *$ & $0.548 * * *$ & $0.556 * * *$ \\
\hline approvalRate & 0.063 & 0.051 & 0.063 & 0.077 & 0.062 & 0.110 & 0.069 & 0.074 & 0.077 \\
\hline regDays & $-4.375 * * *$ & $-4.41 * * *$ & $-4.358 * * *$ & $-4.374 * * *$ & $-4.399^{* * *}$ & $-4.324 * * *$ & $-4.400 * * *$ & $-4.345 * * *$ & $-4.337 * * *$ \\
\hline $\log ($ friends $)$ & $-0.088 *$ & $-0.084 *$ & $-0.086^{*}$ & $-0.086^{*}$ & $-0.079^{*}$ & -0.064 & $-0.087 *$ & $-0.072 *$ & -0.069 \\
\hline $\log ($ referralRwd $)$ & -0.015 & -0.017 & -0.017 & -0.015 & -0.016 & -0.016 & -0.014 & $-0.017 *$ & $-0.018 *$ \\
\hline times & $-0.142 * *$ & $-0.142 * *$ & $-0.147 * *$ & $-0.142 * *$ & $-0.139 * *$ & $-0.139 * *$ & $-0.144 * *$ & $-0.144 * *$ & $-0.146^{* *}$ \\
\hline \multicolumn{10}{|l|}{ Outcome } \\
\hline $\log$ (gain) & -0.039 & -0.011 & -0.061 & -0.037 & 0.002 & -0.088 & -0.042 & -0.072 & -0.004 \\
\hline $\log (\operatorname{loss})$ & 0.022 & 0.146 & 0.095 & 0.026 & 0.180 . & 0.067 & 0.015 & $0.162 * *$ & -0.001 \\
\hline $\log (\operatorname{avgRwd})$ & -0.092 & 0.174 & 0.333 & -0.101 & 0.140 & 0.345 & -0.065 & -0.093 & $0.770 * * *$ \\
\hline $\log$ (followers) & 0.221 & -0.039 & -0.195 & 0.223 & -0.043 & -0.177 & $0.216^{*}$ & -0.028 & $-0.161 *$ \\
\hline verifiedRatio & 0.652 & 1.105 & 0.989 & 0.650 & 1.097. & 0.971 & 0.622 & 0.737 . & 0.000 \\
\hline $\log ($ tweetNum) & 0.001 & $-0.478 * * *$ & $-0.252 *$ & 0.000 & $-0.396 * * *$ & $-0.260 *$ & -0.014 & $-0.409 * * *$ & $-0.463 * * *$ \\
\hline $\log ($ taskNum) & $-0.379 * * *$ & $-0.483 * * *$ & $-0.570 * * *$ & $-0.378 * * *$ & $-0.520 * * *$ & $-0.574 * * *$ & $-0.369 * * *$ & $-0.466 * * *$ & $-0.778 * * *$ \\
\hline approvalRate & 0.704 & 1.708. & -0.300 & 0.706 & 1.707. & -0.338 & 0.672 & -0.409 & $1.119^{*}$ \\
\hline regDays & 0.102 & $2.826^{*}$ & $3.258 * *$ & 0.101 & $2.784^{*}$ & $3.242 * *$ & 0.124 & $3.692 * * *$ & $4.334 * * *$ \\
\hline $\log ($ friends $)$ & $0.420 * *$ & 0.131 & 0.368 & $0.422 * *$ & 0.065 & 0.358 & $0.419 * *$ & $-0.432 * *$ & 0.287 . \\
\hline $\log ($ referralRwd $)$ & 0.023 & 0.003 & -0.095 & 0.021 & -0.014 & -0.092 & 0.021 & $0.101 * *$ & $-0.204 * * *$ \\
\hline times & -0.285 & -0.102 & 0.017 & -0.285 & -0.092 & 0.020 & -0.276 & 0.102 & $1.009 *$ \\
\hline \multicolumn{10}{|l|}{ Heterogeneity } \\
\hline$\delta$ (selection) & $1.310 * * *$ & $1.315 * * *$ & $1.314 * * *$ & $1.31 * * *$ & $1.308^{* * * *}$ & $1.277^{* * * *}$ & $1.311 * * *$ & $1.290 * * *$ & $1.291 * * *$ \\
\hline$\sigma$ (outcome) & $1.609 * * *$ & $2.190 * * *$ & $2.693 * * *$ & $1.607 * * *$ & $2.132 * * *$ & $2.616^{* * * *}$ & $1.592 * * *$ & $2.106 * * *$ & $2.370 * * *$ \\
\hline$\gamma$ (outcome) & 0.097 & $1.131 * * *$ & $1.371 * *$ & 0.094 & $1.123 * * *$ & $1.315^{* * * *}$ & & & \\
\hline \multicolumn{10}{|l|}{ Correlation } \\
\hline$\rho$ (endorser) & $-0.216 * * *$ & $-0.253 * * *$ & $-0.247 * * *$ & $-0.212 * * *$ & $-0.211 * * *$ & $-0.145^{* *}$ & $-0.213 * * *$ & $-0.117 * *$ & $-0.235 * * *$ \\
\hline$\tau$ (endorser-task) & 0.008 & 0.199 & 0.312 . & & & & & & \\
\hline \multicolumn{10}{|l|}{ Fitness } \\
\hline Log Likelihood & -7229.7 & -7304.5 & -7216.5 & -7229.7 & -7304.8 & -7219.0 & -7229.5 & -7319.4 & -7251.7 \\
\hline AIC & 14583.4 & 14733.0 & 14557.0 & 14581.4 & 14731.5 & 14560.0 & 14579.1 & 14758.8 & 14623.4 \\
\hline BIC & 15224.2 & 15373.8 & 15197.8 & 15211.8 & 15362.0 & 15190.4 & 15199.2 & 15378.9 & 15243.5 \\
\hline
\end{tabular}


Table OA.7 Robustness to Potential Outliers

\begin{tabular}{|c|c|c|c|c|c|c|c|c|c|}
\hline & \multicolumn{3}{|c|}{ All Tasks } & \multicolumn{3}{|c|}{ Without Task 2} & \multicolumn{3}{|c|}{ Without Tasks 1-4 } \\
\hline & likes & comments & retweets & likes & comments & retweets & likes & comments & retweets \\
\hline \multicolumn{10}{|l|}{ Selection } \\
\hline isEligible & $2.724 * * *$ & $2.723 * * *$ & $2.720 * * *$ & $2.776^{* * * *}$ & $2.769 * * *$ & $2.769 * * *$ & $2.924 * * *$ & $2.920 * * *$ & $2.927 * * *$ \\
\hline $\log ($ gain $)$ & 0.007 & 0.008 & 0.009 & 0.001 & 0.003 & 0.002 & 0.005 & 0.006 & 0.004 \\
\hline $\log (\operatorname{loss})$ & $-0.073 * * *$ & $-0.074 * * *$ & $-0.073 * * *$ & $-0.077 * * *$ & $-0.077 * * *$ & $-0.078^{* * *} *$ & $-0.081 * * *$ & $-0.081 * * *$ & $-0.081 * * *$ \\
\hline $\log (\operatorname{avgRwd})$ & 0.034 & 0.036 & 0.037 & 0.032 & 0.029 & 0.035 & 0.046 & 0.045 & 0.044 \\
\hline $\log$ (followers) & $0.083 * * *$ & $0.077 * * *$ & $0.079 * * *$ & $0.085^{* * *}$ & $0.080 * * *$ & $0.079 * * *$ & $0.073 * * *$ & $0.071 * * *$ & $0.068 * * *$ \\
\hline verifiedRatio & $0.256^{*}$ & $0.297 * *$ & $0.283 * *$ & $0.264^{*}$ & $0.313^{* *} *$ & $0.303^{* *} *$ & 0.174 & 0.185 & 0.183 \\
\hline $\log ($ tweetNum) & 0.035 & $0.045^{*}$ & 0.039 & 0.034 & $0.045^{*}$ & 0.041 & 0.037 & 0.040 & 0.038 . \\
\hline $\log ($ taskNum) & $0.555^{* * *}$ & $0.564 * * *$ & $0.561 * * *$ & $0.56^{* * *}$ & $0.572 * * *$ & $0.566^{* * *}$ & $0.596 * * *$ & $0.600 * * *$ & $0.589 * * *$ \\
\hline approvalRate & 0.063 & 0.051 & 0.063 & 0.091 & 0.058 & 0.077 & 0.045 & 0.033 & 0.053 \\
\hline regDays & $-4.375^{* * *}$ & $-4.410^{* * *}$ & $-4.358 * * *$ & $-4.433^{* * *}$ & $-4.479^{* * *}$ & $-4.444 * * *$ & $-4.632 * * *$ & $-4.594 * * *$ & $-4.535^{* * *}$ \\
\hline $\log$ (friends) & $-0.088^{*}$ & $-0.084 *$ & $-0.086^{*}$ & $-0.085^{*}$ & $-0.083^{*}$ & -0.075 & $-0.089 *$ & $-0.084 *$ & $-0.078 *$ \\
\hline $\log ($ referralRwd $)$ & -0.015 & -0.017 & -0.017 & -0.015 & -0.017 & -0.017 & $-0.023 *$ & $-0.024 * *$ & $-0.027 * *$ \\
\hline times & $-0.142 * *$ & $-0.142 * *$ & $-0.147 * *$ & $-0.140^{* *}$ & $-0.134 * *$ & $-0.138^{* *}$ & -0.076 & -0.077 & -0.082 \\
\hline \multicolumn{10}{|l|}{ Outcome } \\
\hline $\log ($ gain $)$ & -0.039 & -0.011 & -0.061 & -0.035 & -0.008 & 0.008 & -0.110 & -0.039 & -0.021 \\
\hline $\log (\operatorname{loss})$ & 0.022 & 0.146 & 0.095 & 0.030 & 0.200 & 0.069 & -0.046 & 0.094 & 0.150 \\
\hline $\log (\operatorname{avgRwd})$ & -0.092 & 0.174 & 0.333 & -0.122 & -0.053 & 0.344 & 0.029 & 0.041 & 0.319 \\
\hline $\log$ (followers) & 0.221 & -0.039 & -0.195 & 0.231 & 0.013 & -0.114 & 0.200 & -0.123 & -0.105 \\
\hline verifiedRatio & 0.652 & 1.105 & 0.989 & 0.692 & 1.172. & 0.557 & 0.499 & 0.718 & 0.749 \\
\hline $\log ($ tweetNum) & 0.001 & $-0.478 * * *$ & $-0.252^{*}$ & 0.000 & $-0.46^{* * *}$ & $-0.455^{* * *}$ & -0.013 & $-0.317 * * *$ & -0.235 \\
\hline $\log ($ taskNum $)$ & $-0.379 * * *$ & $-0.483 * * *$ & $-0.570 * * *$ & -0.378 *** & $-0.436 * * *$ & $-0.432 * *$ & $-0.343 * *$ & $-0.414 * * *$ & $-0.513 * * *$ \\
\hline approvalRate & 0.704 & 1.708 & -0.300 & 0.651 & 1.551 & -0.400 & 0.941 & 1.169 & -0.817 \\
\hline regDays & 0.102 & $2.826^{*}$ & $3.258^{* *}$ & 0.031 & 2.282 & 3.317. & -0.140 & 1.095 & 2.066 \\
\hline $\log$ (friends) & $0.42 * *$ & 0.131 & 0.368 & $0.419 * *$ & -0.021 & 0.337 & $0.385^{*}$ & -0.015 & 0.254 \\
\hline $\log ($ referralRwd $)$ & 0.023 & 0.003 & -0.095 & 0.021 & 0.030 & -0.113 & 0.030 & 0.048 & -0.031 \\
\hline times & -0.285 & -0.102 & 0.017 & -0.287 & -0.111 & 0.059 & -0.243 & -0.350 & -0.176 \\
\hline \multicolumn{10}{|l|}{ Heterogeneity } \\
\hline$\delta$ (selection) & $1.31 * * *$ & $1.315^{* * *}$ & $1.314 * * *$ & $1.322 * * *$ & $1.332 * * *$ & $1.328 * * *$ & $1.368 * * *$ & $1.363^{* * *}$ & $1.346 * * *$ \\
\hline$\sigma$ (outcome) & $1.609 * * *$ & $2.190 * * *$ & $2.693 * * *$ & $1.605^{* * *}$ & $2.282 * * *$ & $2.828 * * *$ & 1.600 *** & $1.663 * * *$ & $2.210 * * *$ \\
\hline$\gamma$ (outcome) & 0.097 & $1.131 * * *$ & $1.371 * *$ & 0.124 & $1.186^{* * *}$ & $1.438^{* *}$ & 0.000 & $1.620 * * *$ & $1.586^{* * *}$ \\
\hline \multicolumn{10}{|l|}{ Correlation } \\
\hline$\rho$ (endorser) & $-0.216^{* * *}$ & $-0.253^{* * *}$ & $-0.247 * * *$ & $-0.213^{* * *}$ & $-0.267 * * *$ & $-0.260 * * *$ & $-0.194 * *$ & $-0.213 * *$ & $-0.138^{*}$ \\
\hline$\tau$ (endorser-task) & 0.008 & 0.199 & 0.312 . & 0.022 & 0.206 & $0.344^{*}$ & 0.000 & 0.058 & 0.094 \\
\hline \multicolumn{10}{|l|}{ Fitness } \\
\hline Log Likelihood & -7229.7 & -7304.5 & -7216.5 & -7168.4 & -7234.5 & -7147.1 & -6543.4 & -6583.6 & -6497.7 \\
\hline AIC & 14583.4 & 14733.0 & 14557.0 & 14460.7 & 14593.0 & 14418.3 & 13202.8 & 13283.2 & 13111.5 \\
\hline $\mathrm{BIC}$ & 15224.2 & 15373.8 & 15197.8 & 15099.6 & 15231.9 & 15057.2 & 13794.9 & 13875.3 & 13703.5 \\
\hline
\end{tabular}




\section{Distribution of Endorsers}

The three plots below show the distribution of the four types of endorsers in Table 12 in a twodimensional space, based on endorsers' effectiveness in generating likes, comments, and retweets, respectively. The $\mathrm{x}$-variable is the logged number of tweets an endorser has posted on the social media platform, and the $y$-variable is the logged number of tasks an endorser has participated on the paid endorsement platform. Both variables are representative characteristics that advertisers can use for targeting. The dots in different colors represent different types of endorsers. The big dots represent the average characteristics of endorsers in each task (i.e., 31 big dots in each color). There are two main findings from these plots.

1) While the plots for comment and retweet are largely similar, the plot for like is quite different. This contrast visually illustrates that, endorsers who are effective in generating likes and endorsers who are effectively in generating comments and retweets have very different characteristics.

2) For the comment and retweet plots, it can be seen that the four types of endorsers roughly locates in four different regions (responsive and effective: topleft; responsive and ineffective: topright; unresponsive and effective: bottomleft; unresponsive and ineffective: bottomright). The separability of endorsers will become even better after considering additional characteristics of endorsers. This means, in practice, it's possible to target different versions of tasks to different types of endorsers based on their characteristics.

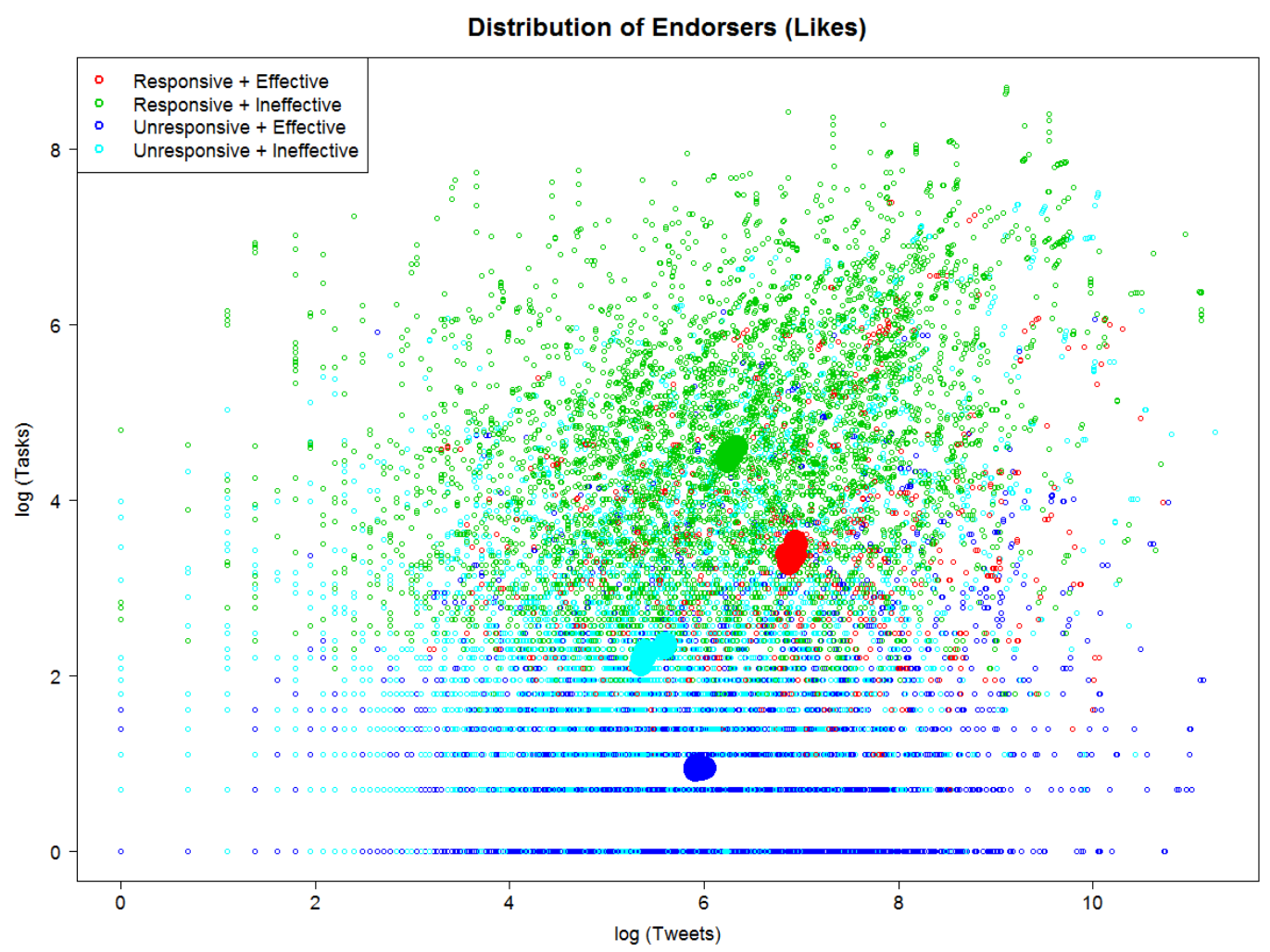



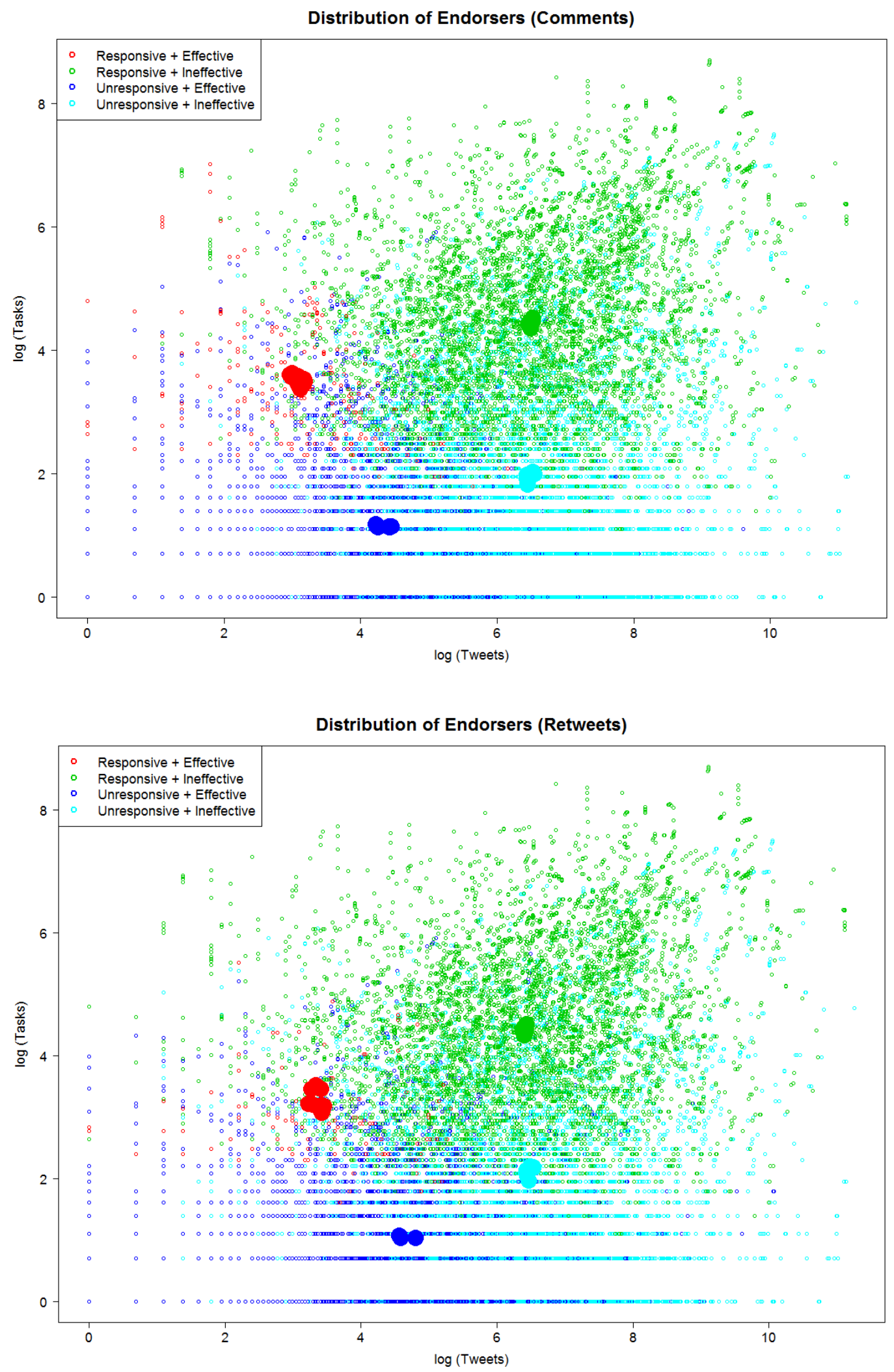